\title{
Near Future Perspectives of QCD Spin Studies
}

\author{
P. M. Nadolsky, S. M. Troshin, N. E. Tyurin \\ Institute for High Energy Physics \\ 142284 Protvino, Moscow Region, Russia
}

\begin{abstract}
We consider the physics motivations and perspectives for the study of spin phenomena at the future high energy accelerators. The possibilities to use the already operating machines are also discussed. It is emphasized that the present status of QCD spin studies necessarily requires wide range of spin measurements.
\end{abstract}

\section{Contents}

Introduction $\quad 2$

1 Spin Studies in Hadronic Reactions 3

1.1 Chiral Invariance and Spin Properties of QCD . . . . . . . . . . . . . 3

1.2 Helicity Properties of Exclusive Processes . . . . . . . . . . . . . . . . 5

1.3 Experiments to Study Spin Effects in Exclusive Reactions . . . . . . . 7

1.4 Tests of QCD in Inclusive Processes . . . . . . . . . . . . . . . . . . . 10

1.4 .1 Higher Twist Contributions . . . . . . . . . . . . . . . . . . . 13

1.4 .2 Experiments to Test QCD in Inclusive Reactions . . . . . . . 14

1.5 Nonperturbative Treatment of Spin Effects . . . . . . . . . . . . . . . 16

$\begin{array}{llr}2 & \text { Studies of Nucleon Spin Structure } & 17\end{array}$

2.1 Deep Inelastic Scattering and Spin Structure of Nucleon . . . . . . . 17

2.2 Experiments to Study Spin Structure of Nucleon . . . . . . . . . . . . 20

2.2 .1 Deep Inelastic Scattering . . . . . . . . . . . . . . . . . . . . 20

2.2 .2 Direct Photon and Jet Production. . . . . . . . . . . . . . . . 24

$2.2 .3 \quad$ Drell-Yan Processes and Polarization of Sea . . . . . . . . . . 25

$2.2 .4 \quad \chi_{\text {-Production Processes }}^{2} \ldots \ldots \ldots \ldots$. . . . . . . . 26

2.2 .5 One-Spin Asymmetry Probe of Parton Spin Densities . . . . . 27

2.3 Transverse Spin Structure of Nucleon . . . . . . . . . . . . . . . . 28

2.3.1 Experiments to Measure the Transverse Spin Quark Densities 33 
3 Spin Phenomena and Electroweak Interactions 35

$4 \quad$ Search for New Particles and Spin Effects 36

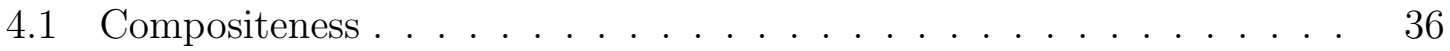

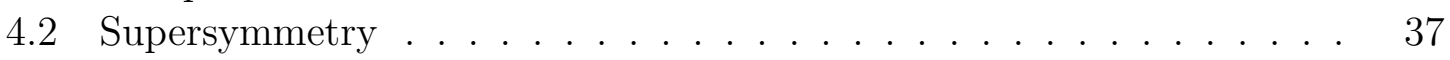

$\begin{array}{lll}5 \text { Spin Effects in } e^{+} e^{-}-\text {Collisions } & 40\end{array}$

\section{Introduction}

The physics of spin effects in particle interactions at large and small distances provides valuable information on the fundamental properties of particles: their wave functions, short distance behavior of the lepton, quark and gluon interactions, mechanisms of chiral symmetry breaking and confinement.

Experiments play the leading role in study of spin phenomena at present and provide a fuel for theoretical analysis. The experiments to study spin phenomena are foreseen at almost all new accelerator facilities. By this time few new accelerators - colliders, have been designed. These are the SSC, proton-proton superconducting supercollider with the beam energy $20 \mathrm{TeV}$ under construction now and the protonproton large collider (LHC) with the beam energy $8 \mathrm{TeV}$ designed at CERN. The UNK , a fixed target machine with the energy $0.6 \mathrm{TeV}$ at first stage is under construction at IHEP, Serpukhov. Here a wide range of spin studies in hadronic reactions is planned in experiments with internal polarized jet target.

It is very essential for spin experiments that Siberian snake concept makes the real possibility to have accelerated polarized beams []]. There are plans to have accelerated polarized beam at Fermilab Main Injector and Tevatron-Collider [2] and these studies were commissioned by Fermilab. Collisions of polarized proton beam with unpolarized antiproton beam at $\sqrt{s}=2 \mathrm{TeV}$ could be realized at TevatronCollider and this will allow to test spin properties of QCD at highest energy in various hadronic processes.

Acceleration of polarized protons up to $2 \mathrm{TeV}$ could be realized at SSC.

There are plans to have the polarized proton beams at Relativistic Heavy Ions Collider (RHIC, BNL) [3]. Proposal on spin physics at RHIC [4] has been initially approved.

At DESY the $e p$-collider (HERA) with the energy of electrons $35 \mathrm{GeV}$ and the proton energy $820 \mathrm{GeV}$ (most probably the proton beam energy will be raised up to 1 $\mathrm{TeV}$ ) is already in operation. Experiment HERMES devoted to study spin structure functions in deep inelastic scattering [5] also has been approved. It will provide data on the structure of nucleons and the tests of QCD, in particular, Bjorken sum rule.

At CERN the large electron-positron collider LEP with the energy $50 \times 50 \mathrm{GeV}$ is operating. The LEP-200 project is underway. Following the known EMC experiment 
the new SMC experiment repeated the measurements of the proton spin structure function performed earlier by the EMC group at SPS but with better accuracy.

The first linear electron-positron collider (SLC) started to operate recently at SLAC with polarized beams. The first exciting results on spin structure of neutron have been obtained. Construction of the linear electron-positron colliders with the center-of-mass energy from 0.5 to $2 \mathrm{TeV}$ is now being worked out also.

The main goal of these facilities is exploration of the energy range, characterized by the scale of $1 \mathrm{TeV}$. It is expected that the experiments would reveal a spectrum of new phenomena related to the Higgs boson, new gauge bosons and supersymmetric particles, manifestation of the compositeness of leptons and quarks and possible mechanisms of mass generation.

Of course, there is a chance that some of new phenomena will be found without measurements of the spin observables. However, the polarization measurements provide additional opportunities to detect new physics. Moreover, these measurements are absolutely necessary to study chiral structure of the new particles couplings.

We review here the possibilities to study hadron dynamics with the help of spin effects as the closest perspectives in the field. Also the perspectives related to electroweak interactions and new physics are mentioned.

\section{Spin Studies in Hadronic Reactions}

\subsection{Chiral Invariance and Spin Properties of QCD}

Nowadays Quantum Chromodynamics (QCD) is generally accepted as a theory of strong interaction. The perturbative expansion based on the asymptotic freedom of QCD allows one to calculate the observables in hard processes and apply this theory to the world of particle interactions. The QCD lagrangian has the form

$$
\mathcal{L}_{Q C D}=\bar{\psi}(x)(i \hat{D}-m) \psi(x)-\frac{1}{4} \operatorname{tr}\left(G_{\mu \nu} G^{\mu \nu}\right),
$$

where the covariant derivative

$$
\hat{D}=\gamma^{\mu} D_{\mu} \quad D_{\mu}=\partial_{\mu}-i g \frac{\lambda^{a}}{2} G_{\mu}^{a},
$$

$G_{\mu \nu}^{a}$ is the gluon field strength tensor, $m=\operatorname{diag}\left(m_{u}, m_{d}, m_{s}\right)$ and the matrices $\lambda^{a}$ are the generators of the $S U(3)_{c}$ color group. By construction the $\mathcal{L}_{Q C D}$ is an invariant under local gauge $S U(3)_{c}$ transformations. Contrary to QED this lagrangian describes self-interaction of the massless color gluons and contains the factors trilinear and quadrilinear over the gauge fields $G_{\mu}^{\alpha}$.

Chiral invariance and vector nature of QCD impose the important constraints on spin observables. Current quarks entering the QCD lagrangian have small masses and may be considered as massless objects. This is a good approximation for $u$ - and 
$d$-quarks and sometimes it is also used for $s$-quark. In this case of $N_{f}=3$ the QCD lagrangian is invariant under the chiral transformations of the chiral $S U(3)_{L} \times S U(3)_{R}$ group, i.e. it is invariant under the global transformations

$$
\psi_{L} \rightarrow L \psi_{L}, \quad \psi_{R} \rightarrow R \psi_{R}
$$

where $L$ and $R$ stand for the $S U(3)$ transformations and

$$
\psi_{R}=\Gamma_{R} \psi, \quad \psi_{L}=\Gamma_{L} \psi
$$

The operators

$$
\Gamma_{R}=\frac{1}{2}\left(1+\gamma_{5}\right) \quad \text { and } \quad \Gamma_{L}=\frac{1}{2}\left(1-\gamma_{5}\right)
$$

are the projection operators. $\psi_{L}$ and $\psi_{R}$ are referred as left and right chirality componenets, i.e. the chirality is the eigenvalue of the Dirac $\gamma_{5}$ matrix:

$$
\gamma_{5} \psi_{L}=-\psi_{L}, \quad \gamma_{5} \psi_{R}=\psi_{R} .
$$

QCD interactions are the same for the left and right quarks in the chiral limit $m_{q} \rightarrow 0$ :

$$
\bar{\psi} \hat{D} \psi=\bar{\psi}_{L} \hat{D} \psi_{L}+\bar{\psi}_{R} \hat{D} \psi_{R} .
$$

As a result the left (right)-handed massless particles will always stay left (right) handed ones. Since all the quarks are massless and have the same QCD coupling, there exists a separate $S U(3)$ flavor invariance in right and left worlds. This means invariance under transformation from $S U(3)_{L} \times S U(3)_{R}$ group.

So, perturbative QCD deals with interactions at short distances and perturbative vacuum (invariant under the chiral transformations).

For massless quarks chirality and helicity coincide:

$$
\psi_{1 / 2}=\psi_{R}, \quad \psi_{-1 / 2}=\psi_{L} .
$$

If the quarks have a non-zero masses the above operators $\Gamma_{L}$ and $\Gamma_{R}$ do not yield the helicity precisely. Small mass term in the QCD lagrangian leads also to explicit chiral symmetry breaking. In that case chirality and helicity are equal approximately at high energies, namely:

$$
\psi_{1 / 2}=\psi_{R}+O\left(\frac{m}{\sqrt{\hat{s}}}\right) \psi_{L}, \quad \psi_{-1 / 2}=\psi_{L}+O\left(\frac{m}{\sqrt{\hat{s}}}\right) \psi_{R},
$$

where indices $\pm 1 / 2$ denote the quark helicities.

Thus, any quark line entering Feynman diagram corresponding to the lagrangian Eq. (II) will emerge with unaltered helicity since helicity flip amplitude is proportional to current quark mass. The quark helicity conservation is the most characteristic feature of perturbative theory with vector coupling. For instance, tensor or pseudoscalar exchange would ensure flip of quark helicity. 
In order to get a nonvanishing polarization it is necessary that the helicity flip amplitude being a non-zero one and in addition the phases of the helicity flip $F_{f}$ and non-flip $F_{n f}$ amplitudes are to be different, since

$$
P \propto \operatorname{Im}\left(F_{n f} F_{f}^{*}\right)
$$

In perturbative QCD the quark helicity flip amplitude is of the order of $m_{q} / \sqrt{\hat{s}}$ [6]. Since the amplitudes are real in the Born approximation, it is necessary at least to consider the diagrams of the fourth order in coupling constant $g$ to get a non-zero imaginary part. Thus, quark helicity flip amplitude will be proportional

$$
F_{f}^{q} \propto \frac{\alpha_{s} m_{q}}{\sqrt{\hat{s}}} F_{n f}^{q}
$$

and polarization has to be vanishingly small in hard interactions (where effective coupling constant is small):

$$
P_{q} \propto \frac{\alpha_{s} m_{q}}{\sqrt{\hat{s}}}
$$

due to large value of $\sqrt{\hat{s}} \sim p_{\perp}$ and small values of $\alpha_{s} m_{q}$, where $m_{q}$ stands for mass of current quark. Even for the top quark with mass $m_{t}=140 \mathrm{GeV}$ the predicted value of transverse polarization is equal to few percents [7].

It should be noted that lattice calculations, low-energy phenomenology and absence of parity doublets in particle spectrum strongly indicate that in the real world the chiral group $S U(3)_{L} \times S U(3)_{R}$ is broken down to $S U(3)_{V}$ with appearance of $N_{f}^{2}-1=8$ Goldstone bosons $(\pi, \mathrm{K}$ and $\eta)$. The dynamical realization of the chiral symmetry breaking in the Nambu-Jona-Lasinio model suggests that the pion, for instance, should be considered as a collective state with strong admixtures of multi $q \bar{q}-$ components.

Therefore, one should note here, that even when the quarks are massless, chirality is not a symmetry of QCD, it is broken (hidden) by the vacuum state, which can be imagined as a complex mixture of virtual quark pairs and is not invariant under the chiral group transformations. This is essentially a non-perturbative effect.

Thus one could expect significant spin effects due to non-perturbative dynamics. However, our primary goal here is to consider the perturbative QCD predictions.

\subsection{Helicity Properties of Exclusive Processes}

Convertion of conclusions at the constituent level into the predictions the predictions for hadron is very complicated problem in exclusive processes. The general recipe is the use of the factorization theorems for exclusive and inclusive processes [8].

It should be noted that even in the leading order, factorization is not a trivial procedure for hadronic processes. Let us turn first to the Brodsky-Lepage approach [9] to factorization in exclusive processes. The important feature of the approach 
is that each parton distribution amplitude is related to a single hadron. This assumption allows to separate bound state dynamics (long distance interactions) from perturbative dynamics of hard parton scattering. The integration over all transverse momenta of the constituents $\left[d^{2} k_{\perp}\right]$ in definition of the distribution amplitude for exclusive processes projects hadron wave function onto the state with $L_{z}=0$ (see, 9] and, e.g. [10], [11]). Hence, hadron helicity is equal to the sum of the valence quark helicities:

$$
\sum_{i=1}^{n} \lambda_{i}=\lambda_{h}
$$

This equation is valid in the framework of the above approach in all orders in $\alpha_{s}\left(Q^{2}\right)$ and in the leading order in $1 / Q$.

The amplitude of constituent interactions preserves the total helicity of the valence quarks with accuracy up to vanishingly small terms of order of $O\left(m_{q} / Q\right)$, where $m_{q}$ is current quark mass. Along with Eq. (7) the latter conclusion leads to the helicity conservation law - the sums of hadron helicities in the initial and final states are equal, i.e. for reaction $A+B \rightarrow C+D[9]$ :

$$
\lambda_{A}+\lambda_{B}=\lambda_{C}+\lambda_{D}
$$

Eq. (8) provides important experimental consequences. The helicity conservation rule results in vanishing of the one-spin asymmetries for hard exclusive processes. For example, the analyzing power in elastic $p p$-scattering is proportional to the amplitude $F_{5}$, which describes the transition

$$
|1 / 2,1 / 2>\rightarrow| 1 / 2,-1 / 2>
$$

between the initial state with helicity 1 and the final state with helicity 0 . Eq. (8) is not satisfied then and

$$
F_{5}=0
$$

The Brodsky-Lepage helicity conservation rule provides unambiguous predictions for the elastic $p p$-scattering at large angles. In the leading order:

$$
A=P=A_{s l}=0, \quad \text { and } \quad A_{n n}=-A_{s s} .
$$

This prediction is in disagreement with the available experimental data indicating increase of the analyzing power with the momentum transfer.

However, the above hard scattering picture meets with certain complications of the above hard scattering picture. The most significant ones are associated with independent scatterings of quarks [12]. Such a picture demands that all three separate scatterings rather than one are to be hard. In the framework of this Landshoff mechanism quarks before the collision are in the state with relative transverse distance $\Delta b \sim 1$ Fermi and suffer independent scatterings at the same angle. The relevant amplitude corresponds to the independent quark scatterings. For $p p$-scattering the 
independent scattering mechanism is determined by the three independent processes of $q q$-scattering at the same angle $\theta_{i} \sim \theta_{c . m}$. These three quark scatterings take place along the normal to the scattering plane. Simple factorization of small and large distances does not take place here.

Comprehensive studies of the both hard scattering mechanisms for hard exclusive processes indicate that the quark independent scattering dominates at very high energies [13], [14].

The quark independent scattering has no rotational invariance and therefore involves the states with non-zero orbital momentum [14], [11]. Orbital momentum may be transformed into spin of the hadron at long-distance evolution and therefore helicity conservation will not take place for the hadron scattering while it does for the quark scattering. This approach allow the hadron helicity flip without flip of a quark helicity and provides a hope that the observed large spin effects would be explained within perturbative QCD.

\subsection{Experiments to Study Spin Effects in Exclusive Reac- tions}

In the nearest perspective the spin phenomena studies are expected to be carried out at the fixed target facilities. Of the primary interest here are the measurements of the spin observables in the high-energy elastic scattering at high $p_{\perp}^{2}$. Such measurements are planned, e.g. at the UNK with the use of internal polarized jet target (NEPTUNA experiment) [15 at the energy range $400-3000 \mathrm{GeV}$.

General arguments and comparison with experimental data show that power law fall-off of differential cross-sections is valid at quite low momentum transfers. For example, fixed $\theta_{c . m}$. scaling for elastic $p p$-scattering is in agreement with experimental data starting at $\sqrt{s}=5 \mathrm{GeV}$ and $\theta_{c . m}$. $\simeq 40^{\circ}$, i. e. for $p_{\perp}^{2}=2-3(\mathrm{GeV} / \mathrm{c})^{2}$. The measurements of the analyzing power and the spin-spin correlation parameters at large $p_{\perp}^{2}$ values were performed in the 10 to $30 \mathrm{GeV}$ laboratory energy region. The experiments unambiguously demonstrated that the relations (9) are violated [16]. Large one-spin asymmetry $A$ observed at $p_{L}=28 \mathrm{GeV} / \mathrm{c}$ and high $p_{\perp}^{2}$ reveals pronounced tendency (Fig. 1) to rise with $p_{\perp}^{2}$ and it reaches $24 \%$ at $p_{\perp}^{2}=6.5$ $(\mathrm{GeV} / \mathrm{c})^{2}$.

The main goal of the NEPTUN-A experiment is to determine if the unexpected large values of $A$ found in the proton-proton elastic scattering at the AGS persist to the energies of hundreds GeV. Besides that the NEPTUN program stipulates the studies of [17]:

- asymmetries in the inclusive production of charged and neutral mesons, as well as the photon production in hard interactions on a polarized target;

- asymmetries in the production of jets and lepton pairs; 
Figure 1: The analyzing power, $A$ is plotted against $p_{\perp}^{2}$ for polarized $p p$ elastic scattering at 24 and $28 \mathrm{GeV} / \mathrm{c}$. 
- polarization in the inclusive production of hyperons, the spin transfer parameter.

This program should allow to make definite conclusion on validity of perturbative QCD due to exploration of the new energy region where contributions of the higher twists are expected to be small at high $p_{\perp}^{2}$. The experiment will also collect the data on the nucleon spin structure. We will discuss these issue more thoroughly below. The experiment is intended to run with the use of the unpolarized proton beam at the UNK starting from energy $400 \mathrm{GeV}$ and later at $3 \mathrm{TeV}$.

It seems important to clarify what kind of hard scattering (independent quark scattering, Brodsky-Lepage mechanism or non-perturbative quark interaction) gives the main contribution to hard scattering. It would be desirable to answer when the experiments would reveal analyzing power at high energies. Valuable information in that direction could be gained from nuclear target experiments. In these experiments a nuclear target serves like a filter to eliminate components of hadron wave function with large transverse separation between the quarks [18]. The measurements of nuclear analyzing power for such components should reveal attenuation of the analyzing power to zero for a nucleus with critical atomic number. The analyzing power would stay zero beyond that number. and above that number analyzing power would stay zero [18. However, if the analyzing power still persists for nuclei with atomic numbers above the critical one then one should arrive to conclusion on a non-perturbative origin of spin effects in hard scattering. Thus, the nuclear target experiments are very useful to discriminate various hard scattering models.

An interesting proposal has been done recently by the SPIN collaboration. It was suggested to upgrade the Tevatron (FNAL) to get the accelerated polarized proton beam [2]. The proposal also includes acceleration of polarized protons to 120 and 150 $\mathrm{GeV}$ in the Fermilab Main Injector and the study of the spin-spin parameter $A_{n n}$ and the spin-orbit asymmetry $A$ in proton-proton elastic scattering at high values of $p_{\perp}^{2}$. The design of the polarized proton capability for the Tevatron-Collider has been commissioned as a further development to study wide range of spin phenomena at Fermilab.

The primary physics goal of the SPIN is to determine if large spin-spin forces revealed by the previous $A_{n n}$ measurements persist at the energies as high as 120 $\mathrm{GeV}$. There is also possibility to measure one-spin asymmetry $A$. In that respect this study would extend the NEPTUN-A program at the UNK to cover the energy range close to $100 \mathrm{GeV}$.

Polarized proton beam of high intensity is an essential tool to measure the analyzing power and spin-spin correlation parameters at high- $p_{\perp}^{2}$ values. It seems important to proceed with these measurements in the region of hundreds $\mathrm{GeV}$ first to trace the experimental discoveries of the tens $\mathrm{GeV}$ region. It is also important to carry out measurements of spin parameters in the wide range of hard exclusive reactions in the region of the tens of $\mathrm{GeV}$ (experiment EVA at Brookhaven). 


\subsection{Tests of QCD in Inclusive Processes}

There are wide opportunities for the use of accelerated polarized beam at the TevatronCollider. Results from SLAC and CERN (EMC) on the proton spin structure of the proton together with the significant spin effects observed in hard elastic and inclusive processes (Figs. 1-3) show that further experimental studies at TeV colliders should be given a high priority. Such experiments at the Tevatron-Collider with a polarized proton beam will both probe the fundamental couplings of the lagrangian and investigate the proton spin structure.

The polarized proton-antiproton collisions at $\sqrt{s}=2 \mathrm{TeV}$ would provide the new unique opportunities to study spin phenomena at the highest energy and allow to test the Standard Model as well as to search for the new physics beyond the Standard Model. Moreover in the fixed target mode with the use of polarized target the additional availability of polarized proton beam will allow to measure a broad spectrum of the two-spin asymmetries at $p_{L}=1 \mathrm{TeV}$.

One of the main goals of these studies should be the tests of QCD. If the spin densities are known, the factorization theorem allows to calculate the cross sections of hard processes and corresponding one- or two-spin asymmetries in the leading order for the respective cross section differencies (constituent level):

- for one-spin asymmetry, when the particle $A$ is polarized

$$
A_{Y} \sigma^{A+B \rightarrow C+X}=\sum_{a, b, c, d} \int \Delta_{Y} G_{A}^{a} G_{B}^{b} D_{c}^{C} \Delta_{Y} \sigma^{a+b \rightarrow c+d}
$$

- for two-spin asymmetry, when the particles $A$ and $B$ are polarized

$$
A_{Y Y} \sigma^{A+B \rightarrow C+X}=\sum_{a, b, c, d} \int \Delta_{Y} G_{A}^{a} \Delta_{Y} G_{B}^{b} D_{c}^{C} \Delta_{Y} \sigma^{a+b \rightarrow c+d},
$$

- for two-spin asymmetry, when the particle $A$ is polarized and spin state of the particle $C$ is measured

$$
D_{Y Y} \sigma^{A+B \rightarrow C+X}=\sum_{a, b, c, d} \int \Delta_{Y} G_{A}^{a} G_{B}^{b} \Delta_{Y} D_{c}^{C} \Delta_{Y} \sigma^{a+b \rightarrow c+d} .
$$

Here $\Delta_{Y}$ denotes differences of the corresponding quantities for the different orientations $(Y=N$ (transversal) or $L$ (longitudinal)) of single or both spins for the initial or final particles. The function $G_{A}^{a}$ is the density of constituent $a$ in the hadron $A$.

When there are no other vectors measured in experiment besides spin of one particle and momenta of initial particles $A$ and $B$ and final particle $C$ then the parity conservation of strong interactions implies, that one-spin longitudinal asymmetries must be zero, since the spin is an axial vector. Indeed, spin vector of the polarized particle should be contracted with another axial vector. But the only axial vector in 
Figure 2: Spin-spin correlation parameter $A_{n n}$ for fixed c. m. s. angle $\left(90^{0}\right)$ plotted against $p_{l a b}$ 
Figure 3: The polarization of $\Lambda$-hyperons produced by $400 \mathrm{GeV}$ protons plotted against $p_{\perp}$. 
the case is a normal to the scattering plane. Therefore, the only possible asymmetry is connected with the transverse component of the initial particle spin vector.

Since the factorization theorems are valid for the transversely polarized hadrons the definite conclusion with respect to the one-spin transverse asymmetry in the leading order may be obtained. Because of vanishing asymmetry in a hard subprocess

$$
a \propto \alpha_{s} \frac{m_{q}}{\sqrt{s^{\prime}}}
$$

the one-spin transverse asymmetry $A_{N}$ for the hadron process should also vanish. For example, the asymmetry $A_{N} \simeq 0$ in the leading order in the reactions

$$
\pi^{-}+p_{\uparrow} \rightarrow \pi^{0}+X \quad \text { and } \quad p_{\uparrow}+p \rightarrow \pi^{0}+X
$$

since at the constituent level $a \simeq 0$. This should be also true for polarization of $\Lambda$-hyperons in the process

$$
p+p \rightarrow \Lambda_{\uparrow}+X
$$

$P_{\Lambda} \simeq 0$, since polarization of quark appears to be $P_{q} \simeq 0$. The experimental data do not follow these predictions at least up to the transverse momentum values $p_{\perp} \leq 3.5$ $\mathrm{GeV} / c$.

\subsubsection{Higher Twist Contributions}

One of the possible escapes is to assume that the transverse momenta values close to $p_{\perp} \simeq 4 \mathrm{GeV} / c$ are too small the leading order calculations to be reliable. In that case one tries to account for the confinement related effects such as higher twists contributions, presence of diquarks in the hadrons, interactions in the final state, etc. At present time all these effects may be taken into account via model approaches only. However, considerations of the higher twist contributions in the framework of perturbative QCD require a minimal number of model assumptions. For the first time such contributions were used for the transverse asymmetry considerations in paper [19]. In the recent paper [20] the higher twist effect were considered with the use of the generalized factorization theorem [8]. There was calculated the one-spin transverse spin asymmetry in the leading order for the direct-photon production process

$$
p_{\uparrow}+p \rightarrow \gamma+X
$$

at large transverse momentum of the photon. These calculations are based on the consideration of the twist-3 matrix element $T\left(x, s_{T}\right)$ of quark and gluon fields. The account for the quantity $T\left(x, s_{T}\right)$ [20] implies presence of the correlations between the quark fields and the strength of gluonic fields and thus assumes the interaction between quarks and color fields. Therefore, simple parton interpretation disappears. Here an important role belongs to the orbital angular momentum. The asymmetry

$$
A\left(s_{T}, x_{F}, l_{\perp}\right)=E_{l} \frac{d \Delta \sigma_{T}}{d^{3} l} / E_{l} \frac{d \sigma}{d^{3} l},
$$




$$
\Delta \sigma_{T} \equiv \frac{1}{2}\left[\sigma\left(s_{T}, l\right)-\sigma\left(-s_{T}, l\right)\right],
$$

where $l$ is the photon momentum, may be expressed in terms of the parton densities, the matrix element $T\left(x, s_{T}\right)$ and the hard scattering functions accordingly to the generalized factorization theorem [8]. The model assumptions are needed for the $T\left(x, s_{T}\right)$ as well as for quark and gluon spin densities. It was proposed [20] to model the quantity $T\left(x, s_{T}\right)$ with a mass scale times dimensional functions of $x$ :

$$
T\left(x, s_{T}\right) \cong 0.2 F_{2}(x) / x \quad \mathrm{GeV}
$$

or

$$
T\left(x, s_{T}\right) \cong 0.2 F_{2}(x) \quad \mathrm{GeV},
$$

where $0.2 \mathrm{GeV}$ is a mass scale and $F_{2}$ is the structure function measured in the unpolarized deep-inelastic scattering. For the both choices of $T\left(x, s_{T}\right)$ the asymmetry rises to over $20 \%$ as $x_{F}$ approaches 0.8 (for $\sqrt{s}=30 \mathrm{GeV}$ and $l_{\perp}=4 \mathrm{GeV} / \mathrm{c}$ ). This effect originates from the variation with $x$ of the function $x \frac{\partial}{\partial x} T\left(x, s_{T}\right)$ entering the expression for the cross-section.

It was shown [21] that at large negative $x_{F}$ values the twist-three pure gluon correlations become dominant in the direct photon production on the transversely polarized nucleon.

Of course, the essential issue to obtain a non-vanishing one-spin asymmetries is the presence of non-zero relative phase between the helicity flip and non-flip amplitudes. The recently proposed new QCD production mechanism for hard processes at large $x$ [22] leads to the relative phase due to imaginary part of the diagrams with intrinsic $q \bar{q}$-pairs. The quarks in the initial Fock states with such pairs are localized in the region of a small transverse size and therefore they have large transverse momenta.

These results are promising ones and allow to hope that the large transverse asymmetries at large $x_{F}$ observed at the existing facilities (at moderate energies) can be explained within QCD as the higher-twist effects. However, some model assumptions are still needed, in particular, for the choice of the form of higher twist contributions. Thus, the experimental studies of spin effects at large $x_{F}$ values will help to clarify the particular dependence of the higher twists expected to contribute at moderate energies and $Q^{2}$ values.

\subsubsection{Experiments to Test QCD in Inclusive Reactions}

However, the general statement, that in the leading order the one-spin transverse asymmetries at the constituent level are vanishingly small due to vector type of the gluon interaction in QCD is unchanged. Therefore, once the energy and transverse momentum are high enough to rely on the perturbative expansion in QCD and to neglect the higher-twist contributions one should expect $A_{N}=0$. Any violation of this prediction would indicate the necessity of serious modifications of perturbative 
QCD and account for non-perturbative effects. Such helicity conservation violation could be attributed to the chiral symmetry breakdown by the physical vacuum, since the spin properties are intimately tied to the chiral structure of the theory.

The energy independence of large polarization of $\Lambda$-hyperons observed in the region between 12 and $2000 \mathrm{GeV}$ strongly indicates that spin effects persist at high energies. The use of the polarized target and polarized beam will allow to measure the two-spin correlations in the hyperon production at $\mathrm{TeV}$ energies and to reveal the underlying mechanism leading to this still unexplained result.

Moreover, the measurements of the longitudinal asymmetries will provide the data on the production mechanism and on the hadron spin structure. It seems very promising to consider the reactions with weakly decaying baryons in the final state such as

$$
p_{\rightarrow}+\bar{p} \rightarrow \Lambda_{\rightarrow}+X
$$

and to measure the parameter

$$
D_{L L}=\frac{\left.E_{C} \frac{d \sigma}{d^{3} p_{C}}\right|_{\rightrightarrows}-\left.E_{C} \frac{d \sigma}{d^{3} p_{C}}\right|_{\rightleftarrows}}{\left.E_{C} \frac{d \sigma}{d^{3} p_{C}}\right|_{\rightrightarrows}+\left.E_{C} \frac{d \sigma}{d^{3} p_{C}}\right|_{\rightleftarrows}}
$$

where the lower arrow denotes the longitudinal polarization of $\Lambda$-hyperon in the final state. For such a reaction, the subprocess $q \bar{q} \rightarrow s \bar{s}$ will dominate at high $x_{\perp}$ values, while the gluon annihilation $g g \rightarrow s \bar{s}$ will be important at small $x_{\perp}$ values. Therefore this process is quite sensitive to the quark and gluon polarizations inside a proton. Asymmetries at the constituent level are calculable in the framework of perturbative QCD [23]; therefore measurements of the asymmetries $D_{L L}$ will provide the data on quark and gluon spin densities. The theoretical estimations provide rather significant values for $D_{L L}$ at the level of $50 \%$ [24] that cause no problems in the $D_{L L}$ experimental measurements. The measurements of $D_{L L}$ in the fragmentation region at large $x_{F}$ seem to be interesting also from the point of view of the polarization of strange sea and strangeness content of a nucleon. Strange sea may have a large negative polarization accordingly to the SLAC and EMC data interpretations and it should be revealed in $D_{L L}$ analysis.

The problem of the nucleon spin structure studies will be considered further.

The experimental results on elastic scattering as well as results on the inclusive processes support the energy persistence of spin effects. As it was mentioned there is a chance to relate these spin effects to manifestations of the higher-twist terms (quark-gluon and gluon-gluon correlations) [20] [21]. Therefore, the experimental measurements of $A_{N}$ in the relevant kinematical region would reveal the size of the quark-gluon and pure gluonic correlations. However, it could be expected that at the Tevatron-Collider energy $\sqrt{s}=2 \mathrm{TeV}$ the higher-twist terms can be safely neglected at large $p_{\perp}$ values. At such energies the $A_{N}$ measurements should allow a clear test of perturbative QCD in such processes as:

$$
p_{\uparrow}+\bar{p} \rightarrow \pi, \gamma+X,
$$


or

$$
p_{\uparrow}+\bar{p} \rightarrow j e t+X
$$

at large $p_{\perp}$ values.

The measurements of $A_{N}$ in the above processes would also test the chiral structure of the effective lagrangian approaches in the framework of non-perturbative realization of QCD.

\subsection{Nonperturbative Treatment of Spin Effects}

The data on the unexpected spin effects at small distances stimulated development of the model approaches. There was proposed a number of models to describe the spin phenomena. Some of the models use the methods and ideas of QCD, but the other ones are only inspired by QCD. It should be emphasized that description of the spin effects at large angles and in particular of the behavior of the one-spin asymmetries represent a complicated problem not only for perturbative QCD but for the most of the QCD-based models also. If the present trends in the experimental data on the one-spin asymmetries will persist at higher energies it could suggest a non-perturbative origin of spin dynamics and will certainly initiate the further development of the corresponding models.

As it was already mentioned there are two non-perturbative phenomena implied by QCD, namely, the confinement and spontaneous chiral symmetry breakdown. As it currently believed, these phenomena are characterized by the different scales.

It seems, that the crucial role under study of the spin effects belongs to the chiral symmetry breaking. The chiral symmetry breaking means that the chiral symmetry of the QCD lagrangian is hidden by the complicated structure of the QCD vacuum. Due to the chiral symmetry breakdown the hadron structure already at the distance of tenth of Fermi diverges from the parton model picture. The chiral symmetry breaking results in generation of quark masses comparable with the hadron mass scale. Therefore a hadron can be represented as a loosely bounded system of the constituent quarks. The above observations related to the hadron structure lead to understanding of several regularities observed in hadron interactions at large distances. This picture provides also reasonable values for the static characteristics of hadrons, for instance, for their magnetic moments [25].

Generally speaking the significant spin effects observed in hard processes over a long time pointed out a non-perturbative nature of hadron dynamics, in particular, at rather short distances. The non-perturbative dynamics at short distances has been also revealed under the hadron structure studies in deep-inelastic scattering. The chiral models appear to provide a transparent explanation of the results of the spin structure function $g_{1}(x)$ measurements in deep-inelastic scattering of polarized muons on polarized proton target [26]. We return to that important issue in the next section. 
The usefulness of the models based on the chiral symmetry breaking naturally leads to the aspiration to extend the domain of their applicability to the region of small distances [27.

The attempts to incorporate some of the chiral model ideas in a context of the unified approach to a simultaneous description of hadron scattering both at large and small distances were made recently. One should remember that the theoretical approaches to description of hadron phenomena are usually aimed for soft and hard interactions separately. The above mentioned considerations are based on the method of three-dimensional dynamical equations for the scattering amplitude in Quantum Field Theory and on the current ideas on the hadron structure and interaction of the constituents [28]. This approach allows one to describe a number of spin effects. It also predicts:

- non-vanishing one-spin asymmetry in elastic scattering at large angles at asymptotical energies;

- oscillating dependence of the spin-spin correlation parameters in elastic $p p$ scattering in the region of hundreds $\mathrm{GeV}$;

- non-vanishing with energy and oscillating with $p_{\perp}$ polarization in the hyperon production at large $p_{\perp}$ values and $x_{F}$ close to 1 ;

- decrease with energy growth of the asymmetry in the pion production in the central region.

Here we will not discuss further here the model approaches. It is to be noted only that the models are very sensitive to the spin observables and the new results will allow to discriminate various approaches.

\section{Studies of Nucleon Spin Structure}

\subsection{Deep Inelastic Scattering and Spin Structure of Nucleon}

Historically, the deep inelastic scattering of electrons indicated first the presence of point-like constituents in a nucleon. The spin dependent structure functions can be measured in the deep inelastic scattering experiments with polarized lepton beam and a nucleon target. These functions allow one to extract the data on the nucleon's parton distributions which characterize nucleon properties in hard scattering processes. Measurements of the structure functions have provided important results on the quark-gluon structure of hadrons. The spin-independent quark and gluon distributions were measured with high accuracy during twenty years. This is not the case for the spin-dependent structure functions. The longitudinal spin-dependent structure function has been measured at SLAC and CERN. 
The two spin structure functions $G_{1}$ and $G_{2}$ enter the expression for the antisymmetric part of the hadronic tensor $W_{\mu \nu}^{[A]}(p, q, s)$ :

$$
W_{\mu \nu}^{[A]}=\frac{1}{M} \varepsilon_{\mu \nu \lambda \sigma} q^{\lambda}\left[M^{2} s^{\sigma} G_{1}\left(\nu, Q^{2}\right)+\left(p \cdot q s^{\sigma}-s \cdot q p^{\sigma}\right) G_{2}\left(\nu, Q^{2}\right)\right] .
$$

In the Bjorken limit ( $\nu$ and $Q^{2} \rightarrow \infty, x \rightarrow$ const) the scaling is to be observed and the functions $G_{1}$ and $G_{2}$ should depend on $x$ only up to the logarithmic corrections, i.e. they obey the relations:

$$
\begin{aligned}
& M^{2} \nu G_{1}\left(\nu, Q^{2}\right) \rightarrow g_{1}(x), \\
& M \nu^{2} G_{2}\left(\nu, Q^{2}\right) \rightarrow g_{2}(x) .
\end{aligned}
$$

The recent data obtained at CERN by the EMC collaboration triggered a great number of theoretical works devoted to the problem of proton spin and resulted in the extended interest in the future experiments. These data are in disagreement with the expectations based on the constituent quark model. Indeed, the use of the $S U(6)$-symmetrical wave function of the proton results in

$$
\Delta u=4 / 3, \quad \Delta d=-1 / 3, \quad \Delta s=0 \quad \text { and } \quad \sum_{q} \Delta q=1 .
$$

The so-called "spin crisis" puzzle emerged from the experimentally obtained almost zero value for the sum

$$
\sum_{q} \Delta q \simeq 0
$$

and significant negative polarization of strange quarks

$$
\Delta s=-0.18 \pm 0.07 .
$$

The significant value of $\Delta s$ results also from the data on elastic $\nu p$-and $\bar{\nu} p$-scattering [29]. Thus, extrapolation to $q^{2}=0$ of the measured values for the axial $Z^{0}-N$ formfactor gives [30]:

$$
\Delta s=-0.15 \pm 0.08 \text {. }
$$

The above unexpected results stimulated a great theoretical activity in the field.

In general, the SLAC and EMC experiments probe the hadron structure at short distances, whereas the constituent quark model is associated with the long range interactions. Of course, one have to expect that the structure of a nucleon at short distances differs from its structure at long distances. Nevertheless, the referred experiments show up that the spin structure of nucleons is far from trivial and deserves thorough consideration.

Shortly after the EMC analysis had been performed, the perturbative QCD interpretation has been proposed. It is based on account for anomalous gluon contribution to the first moment of $g_{1}$ in the frameworks of parton model [31]. In terms of the 
operator product expansion this is related to the non-conservation of the axial-vector current $J_{\mu 0}^{5}$ in QCD (even in the limit of massless quarks) due to the axial anomaly 32]:

$$
\partial^{\mu} J_{\mu 0}^{5}=2 i \sum_{q}^{N_{f}} m_{q} \bar{q} \gamma_{5} q+N_{f} \frac{\alpha_{s}}{4 \pi} G_{\mu \nu}^{a} \tilde{G}_{\mu \nu}^{a},
$$

where the dual $\tilde{G}$ is defined as $\tilde{G}_{\mu \nu}=\frac{1}{2} \varepsilon_{\mu \nu \sigma \rho} G^{\sigma \rho}$ and $G_{\mu \nu}$ is the gluon field strength tensor. It was proposed to redefine the axial-vector current to ensure its conservation in the limit of massless quarks:

$$
\tilde{J}_{\mu 0}^{5}=J_{\mu 0}^{5}-K_{\mu}
$$

where

$$
K_{\mu}=\frac{\alpha_{s} N_{f}}{2 \pi} \varepsilon_{\mu \nu \rho \sigma} G^{a \nu}\left(\partial^{\rho} G^{\rho \sigma}-\frac{1}{3} g f^{a b c} G^{b \rho} G^{c \sigma}\right) .
$$

This approach removes the anomaly contribution from the matrix elements of the axial-vector current and provides a non-zero contribution to the first moment of the structure function $g_{1}^{p}\left(x, Q^{2}\right)$. The matrix element of the difference $J^{5}-K$ is considered to provide the true quark contribution $\sum_{q} \Delta q$ into the proton spin while the quantity measured in experiment should be replaced by $\tilde{\sum}_{q} \Delta q$ according to the equation

$$
\tilde{\sum_{q}} \Delta q=\sum_{q} \Delta q-N_{f} \frac{\alpha_{s}}{2 \pi} \Delta g
$$

If the used gauge does not imply appearance of the unphysical degrees of freedom, then the quantity $\Delta g$ could be interpreted in terms of the gluon densities with helicity parallel $g_{+}(x)$ and antiparallel $g_{-}(x)$ to the nucleon helicity in the infinite momentum frame, i.e.:

$$
\Delta g=\int_{0}^{1} d x\left[g_{+}(x)-g_{-}(x)\right]
$$

In such approach a photon can see the gluon helicity distribution $\Delta g$ because of the axial anomaly resulting from non-conservation of the singlet axial-vector current. The gluonic contribution does not need to be a small correction because $\Delta g$ grows with $Q^{2}$ :

$$
\Delta g\left(Q^{2}\right)=\frac{\alpha_{s}\left(Q_{0}^{2}\right)}{\alpha_{s}\left(Q^{2}\right)} \Delta g\left(Q_{0}^{2}\right)
$$

owing to the evolution equation [33]. It should be noted, however, that the operator $K_{\mu}$, is not gauge invariant and hence it does not appear in the OPE. The quantities entering Eq.23 depend on the renormalization point.

In the sum rule for the proton spin

$$
\frac{1}{2}=\frac{1}{2} \sum_{q} \Delta q+\Delta g+\left\langle L_{z}^{q}\right\rangle+\left\langle L_{z}^{g}\right\rangle
$$


the individual components, except the first term on the right hand side, are not gauge invariants separately [34], [35]. Thus, the problems associated with the ambiguities in the gluon spin interpretation as matrix element of the current $K_{\mu}$ make this interpretation controversial [36].

Moreover, to reconcile the EMC result with the constituent quark model the large value for $\Delta g$ should be assumed: $\Delta g \sim 5$. Such value can hardly be obtained in the models for the hadron spin structure [37]. In the case the large negative orbital angular momentum $(\sim 5)$ due to quarks and (or) gluons is required to compensate the $\Delta g$. This orbital momentum value has to grow logarithmically with $Q^{2}$ since $\Delta g \sim \alpha_{s}^{-1}$. Thus, the anomalous gluon interpretation of the EMC result based on the hard gluonic contribution to the first moment of $g_{1}$ leads to the new problems. Of course, nobody can deny the possibility of final resolution of all these problems, but under present circumstances, alternative approaches to the problem of the proton spin also deserve consideration.

Thus, if $\Delta g=0$ or we consider the values of $\Delta q$, extracted from the SLAC and EMC data as the true quark contributions to the proton spin, then we should conclude, that the strangeness content of the proton, i.e. the matrix element of axialvector current $\left\langle p\left|\bar{s} \gamma_{\mu} \gamma_{5} s\right| p\right\rangle$ has a large value. In other words, the strange sea provide a significant negative contribution to the spin of proton. The other indications on the strangeness content of the proton follow from $\pi N$-scattering at low energies. In particular, from the value of the so-called $\pi N$ sigma term and the observed baryon masses in the first order over $S U(3)$ symmetry breaking the value of the matrix element $\langle p|\bar{s} s| p\rangle$ has been obtained [38]

$$
m_{s}\langle p|\bar{s} s| p\rangle=334 \pm 132 \mathrm{MeV} .
$$

These results have important implications for the proton structure and should stimulate further experiments to study matrix elements of the strange quark operators.

\subsection{Experiments to Study Spin Structure of Nucleon}

The current experimental situation with the measurements of the spin structure functions is far from being complete. Few independent experimental measurements of the structure functions $g_{1}^{p, n}\left(x, Q^{2}\right)$ have been performed. There are no data on the second spin structure functions $g_{2}\left(x, Q^{2}\right)$ of the proton and neutron. In this situation the further experimental measurements should play a crucial role.

\subsubsection{Deep Inelastic Scattering}

In deep inelastic scattering the both structure functions $g_{1}^{p, n}\left(x, Q^{2}\right)$ and $g_{2}^{p, n}\left(x, Q^{2}\right)$ may be evolved in experiments with the use of longitudinally and transversely polarized targets. Such measurements are planned at HERA in the framework of HERMES project [5]. . It is proposed to use the longitudinally polarized electron beam and 
polarized internal gas target. The presumed types of the target materials are hydrogen, deuterium and ${ }^{3} \mathrm{He}$. The electron beam energy should range between 30 and 35 $\mathrm{GeV}$. The luminosity is expected to be varied from 3.5 to $30 \times 10^{31} \mathrm{~cm}^{-2} \cdot s^{-1}$. It will allow to obtain the spin structure functions for protons and neutrons with the high statistical precision.

This experiment will also allow to discriminate the various models simulating the spin structure of the nucleon. The measurements will give the contribution of valence quarks to the spin of the nucleon, since under the simultaneous measurement of the proton and neutron spin structure functions, the contributions of sea quarks and gluons may be excluded. Being realized, the HERMES project will also provide the possibility to check the Bjorken sum rule derived in the framework of QCD with account for the isospin invariance only and without any additional assumptions. This sum rule takes place for the first moment of the difference $g_{1}^{p}-g_{1}^{n}$ [39:

$$
\int_{0}^{1}\left[g_{1}^{p}(x)-g_{1}^{n}(x)\right] d x=\frac{1}{6} g_{A}\left(1-\frac{\alpha_{s}(Q)}{\pi}\right) .
$$

Verification of Eq. 26 will provide an important test of Quantum Chromodynamics.

It is interesting that experiments SMC (Spin Muon Collaboration) at CERN and E142 at SLAC have reported preliminary results on the spin structure of the neutron. They allowed to test the Bjorken sum rule for the first time. The SMC measurements with polarized muon beam and polarized deuteron target support the original EMC conclusions in particular on validity of the Bjorken sum rule. However, E142 with polarized electron beam and helium-3 target reveals a deviation from the Bjorken sum rule (3 sigma) and shows up that more than a half of the proton's spin is due to quarks.

It is noteworthy that the experiments devoted to measurements of the spin structure functions are now recognized to be among the most important ones and they are planned in many laboratories.

In the above discussions we focused on the deep inelastic scattering on polarized protons and considered the structure function $g_{1}\left(x, Q^{2}\right)$ related to the longitudinal polarization of a proton spin with respect to its momentum, i.e.:

$$
g_{L}\left(x, Q^{2}\right)=g_{1}\left(x, Q^{2}\right) .
$$

In the parton model, $g_{1}$ measures the quark helicity density. The second spindependent proton structure function $g_{2}\left(x, Q^{2}\right)$ is related to transverse polarization of a nucleon spin, i.e.:

$$
g_{\perp}\left(x, Q^{2}\right)=g_{1}\left(x, Q^{2}\right)+g_{2}\left(x, Q^{2}\right) .
$$

It has never been measured and only few theoretical results were obtained. The direct application of the parton model orinally used for $g_{L}\left(x, Q^{2}\right)$ to the function $g_{\perp}\left(x, Q^{2}\right)$ results in the following relation:

$$
g_{\perp}\left(x, Q^{2}\right)=\frac{1}{2} \sum_{q} e_{q}^{2}\left(\frac{m_{q}}{x M}\right) \Delta_{\perp} q\left(x, Q^{2}\right),
$$


where $m_{q}$ is the quark mass, $M$ is the proton mass and

$$
\Delta_{\perp} q\left(x, Q^{2}\right)=q_{\uparrow}\left(x, Q^{2}\right)+\bar{q}_{\uparrow}\left(x, Q^{2}\right)-q_{\downarrow}\left(x, Q^{2}\right)-\bar{q}_{\downarrow}\left(x, Q^{2}\right) .
$$

Eq. 29 implies $g_{\perp}\left(x, Q^{2}\right)=0$ if the massless limit is assumed $m_{q} \rightarrow 0$. This fact served as a basis for the statement on the small value of the structure function $g_{\perp}\left(x, Q^{2}\right)$ and for the doubts in the applicability of the parton model to interpretation of the second structure function and to description of the transversely polarized particles.

It should be noted first, that vanishing of $g_{\perp}\left(x, Q^{2}\right)$ does not take place in the parton model as it was mentioned recently in Ref. 40. Indeed, in this model because of the relation $m_{q}=x M$ (where $M$ is the nucleon mass) the trivial zero-mass limit is inconsistent with the non-zero mass of a nucleon in the rest frame. Therefore in Eq. 29 one should set $m_{q}=x M$. In this case there is no reason for the smallness of the function $g_{\perp}\left(x, Q^{2}\right)=0$. Consideration of in the simple parton model with noninteracting partons, i.e. when the hadron is treated as a gas of free quarks, shows 441] that the spin density of quarks does not depend on the angle between the nucleon polarization and its momentum. For that case

$$
\Delta_{\perp} q(x)=\Delta q(x)
$$

Eq. 30, however, is valid for non-interacting quarks only. In the more realistic models Eq. 30 should not take place, in particular, $Q^{2}$-evolution would spoil it.

In general case one could rely on OPE. The analysis based on the OPE does not depend on the type (longitudinal or transversal) of the nucleon polarization. Consideration of the second structure function $g_{2}\left(x, Q^{2}\right)$ may be performed similar to the study of the function $g_{1}\left(x, Q^{2}\right)$ [40. The significant difference, however, between, the two cases is that the function $g_{1}\left(x, Q^{2}\right)$ receives contribution from the twist-two operators only, whereas $g_{2}\left(x, Q^{2}\right)$ gets contributions from the both twist-two and twist-three operators simultaneously. Note, that the simple partonic interpretation is valid for the twist-two operator contribution. An account for such contribution only provides the following relation for the functions $g_{1}$ and $g_{2}$ [42]:

$$
g_{2}\left(x, Q^{2}\right)=\int_{x}^{1} \frac{d y}{y} g_{1}\left(y, Q^{2}\right)-g_{1}\left(x, Q^{2}\right)
$$

or in the other form

$$
\int_{x}^{1} d x x^{J-1}\left[\frac{J-1}{J} g_{1}\left(x, Q^{2}\right)+g_{2}\left(x, Q^{2}\right)\right]=0 .
$$

The sum rule Eq. 31 allows to calculate the function $g_{2}\left(x, Q^{2}\right)$ from the function $g_{1}\left(x^{\prime}, Q^{2}\right)$ at $x^{\prime} \geq x$ in the framework of the parton model with free on-shell partons. However, there are no reasons to neglect the contributions of the twist-three operators and therefore the function $g_{2}$ is to be represented as follows:

$$
g_{2}\left(x, Q^{2}\right)=g_{2}\left(x, Q^{2}\right)^{[2]}+g_{2}\left(x, Q^{2}\right)^{[3]}
$$


where the first term in the right hand side of Eq. 33 is provided by Eq. 31. The twist-three operator contributions $g_{2}\left(x, Q^{2}\right)^{[3]}$ depend on the effects of quark-gluon interactions and quark masses. Due to the chiral symmetry spontaneous breakdown and the confinement, these contributions should be large enough in any realistic model of a nucleon.

In particular, the case of massive off-shell quarks has been studied [43] in the framework of the covariant parton model. The following expressions for the functions $g_{1}\left(x, Q^{2}\right)$ and $g_{2}\left(x, Q^{2}\right)$ were obtained:

$$
\begin{aligned}
& g_{1}\left(x, Q^{2}\right)=\frac{\pi x}{8} \int^{Q^{2}} d k^{2} d k_{\perp}^{2}\left(1-\frac{k^{2}+k_{\perp}^{2}}{x^{2} M^{2}}\right)\left(\frac{x^{2} M^{2}-k^{2}+k_{\perp}^{2}}{x^{2} M^{2}+k^{2}+k_{\perp}^{2}}\right) \tilde{f}\left(x+\frac{k^{2}+k_{\perp}^{2}}{x^{2} M^{2}}, k^{2}\right), \\
& g_{\perp}\left(x, Q^{2}\right) \equiv g_{1}\left(x, Q^{2}\right)+g_{2}\left(x, Q^{2}\right)=\frac{\pi x}{8} \int^{Q^{2}} d k^{2} d k_{\perp}^{2}\left(\frac{k_{\perp}^{2}}{x^{2} M^{2}}\right) \tilde{f}\left(x+\frac{k^{2}+k_{\perp}^{2}}{x^{2} M^{2}}, k^{2}\right),
\end{aligned}
$$

where the function $\tilde{f}$ is determined by the quark densities in the polarized nucleon. From Eq. 35 it follows that the function $g_{\perp}\left(x, Q^{2}\right)$ is related to the mean transverse parton momentum. The appearance of $k_{\perp}^{2}$ in the expressions for the polarized structure functions shows that the deep inelastic scattering processes with polarized beam and target probe the features of the parton model different from those in the deep inelastic scattering of unpolarized particles.

In the framework of operator product analysis of the structure function, the sum rule for the function $g_{2}\left(x, Q^{2}\right)$ can be obtained. This sum rule was derived in Ref. [44] and is known as Burkhardt-Cottingham sum rule

$$
\int_{0}^{1} g_{2}(x)=0 .
$$

It should be stressed that in the parton model the structure function $g_{1}$ measures the quark helicity distribution in a longitudinally polarized nucleon, whereas in transversely polarized nucleon the structure function $g_{\perp}$ measures the average transverse spin for quarks and $g_{\perp}$ is related to the quark-gluon interactions, i.e. to the higher twist terms.

The estimation of importance of the twist-three operators in the framework of MIT bag model has been done in [40]. As it was mentioned there are two types of the twist-three operators: one is related to the quark gluon interactions and the other one to the effect of quark masses. In the bag model the both types of operators contribute to the function $g_{2}(x)$. These contributions and the twist-two operator contribution are comparable in their values.

Thus the experimental measurements of the structure function $g_{2}(x)$ in the deep inelastic scattering processes with polarized particles are particularly important to reveal nonperturbative effects related to the confinement and chiral symmetry breaking. In the parton model the account for the transverse spin-structure function assumes the study of the off-shell partons and their transverse momenta. 


\subsubsection{Direct Photon and Jet Production}

Besides the studies in deep inelastic scattering, the spin structure of the proton as well as the role of the gluon and sea components of spin, may also be investigated in the hadronic collisions. The hadronic processes allow, in particular, to separate the quark and gluon contributions to the proton spin. The EMC result interpretations are based on the hard gluon or sea quark contributions to the structure function $g_{1}$. The first interpretation imply a large gluon contribution which in principle should easily be detected. The sea quark interpretation may be checked in the Drell-Yan processes. The availability of high-energy colliders with polarized proton beams would allow experimenters to carry a number of studies sensitive to the gluon and sea polarization. There are plans to have polarized beams at the colliders [45]. We discuss here some of the experimental opportunities in this connection.

In principle it is expected that the gluons and sea quarks are equally important sources of the proton spin.

There are few processes which allow to obtain the data on the contribution of gluons to the proton spin. It is production of the direct photons with large $p_{\perp}$ values in collision of the longitudinally polarized protons:

$$
p_{\rightarrow}+p_{\rightarrow} \rightarrow \gamma+X .
$$

Eq. 37 is one of the cleanest reactions to study perturbative QCD, since the photon originates from the hard subprocess and therefore there is no other photons resulting from the fragmentation process. The dominant contribution to the direct photon production is due to the quark-gluon Compton subprocess:

$$
q+g \rightarrow \gamma+q .
$$

The asymmetry is given by the expression:

$$
a_{L L}=\frac{s^{\prime 2}-t^{\prime 2}}{s^{\prime 2}+t^{\prime 2}}
$$

The corresponding asymmetry at a hadron level, $A_{L L}$, is determined by the two-spin asymmetry $a_{L L}$ and the longitudinal spin densities of the quarks $\Delta q(x)$ and gluons $\Delta g(x)$ in polarized proton:

$$
A_{L L}=\frac{\sum_{q} \int[d x] \Delta q\left(x_{1}\right) \Delta g\left(x_{2}\right) \frac{s^{\prime 2}-t^{\prime 2}}{s^{2}+t^{\prime 2}} \frac{d \sigma^{\prime}}{d t^{\prime}}+\Delta g\left(x_{1}\right) \Delta q\left(x_{2}\right) \frac{s^{\prime 2}-u^{\prime 2}}{s^{\prime 2}+u^{\prime 2}} \frac{d \sigma^{\prime}}{d u^{\prime}}}{\sum_{q} \int[d x] q\left(x_{1}\right) g\left(x_{2}\right) \frac{d \sigma^{\prime}}{d t^{\prime}}+g\left(x_{1}\right) q\left(x_{2}\right) \frac{d \sigma^{\prime}}{d u^{\prime}}} .
$$

In Eq. 40 the quantities $d \sigma^{\prime} / d t^{\prime}$ and $d \sigma^{\prime} / d u^{\prime}$ are the known unpolarized differential cross-sections of the quark-gluon Compton subprocess. Since contribution of this subprocess to the asymmetry $A_{L L}$ is proportional to the gluon polarization $\Delta g$, the measurements of this quantity would allow to extract the data on the gluon spin 
density provided the quark densities are known from the deep inelastic scattering processes.

The direct photons can also be produced in the quark annihilation subprocess

$$
q+\bar{q} \rightarrow \gamma+g
$$

This subprocess is sensitive to the sea polarization. The respective asymmetry is $a_{L L}=-1$. The statement that the direct photon production is a good probe of the gluon density is based on the observation that numerically the Compton contribution to proton-proton interactions is much larger than the annihilation contribution. It was recently shown that this is true for the case of polarized proton-proton interactions also and, in particular, for the case of longitudinally polarized protons [46].

The measurements of the direct photon production under the collision of polarized protons may be carried out either with the use of fixed target accelerators or colliders with polarized beams.

The gluon component of the proton spin should also manifest itself in the measurements of $A_{L L}^{j e t}$ and $\Delta \sigma_{L}^{\text {jet }}$ in the jet production process:

$$
p_{\rightarrow}+p_{\rightarrow} \rightarrow j e t+X
$$

Contribution of gluons into the quantity $\Delta \sigma_{L}^{\text {jet }}$ has the following form:

$$
\Delta \sigma_{L}^{j e t}(s)=\frac{\pi \alpha_{s}^{2}}{2 s} \sum_{i, j} \int_{x_{1} x_{2}>\xi} d x_{1} d x_{2} \frac{\Delta g_{i}\left(x_{1}\right)}{x_{1}} \frac{\Delta g_{j}\left(x_{2}\right)}{x_{2}} \Delta H_{i j}\left(z^{0}\right),
$$

where $\xi=4\left(p_{\perp}^{0}\right)^{2} / s, \quad z^{0}=\left(1-\xi / x_{1} x_{2}\right)^{1 / 2}, \quad p_{\perp}^{0}$ is the cutoff determined by the boundary of the hard region. The function $\Delta H_{i j}$ stands for the integral of the cross section difference of the two-particle hard subprocesses. The initial gluons are to be in the corresponding helicity states. Due to large values of the gluon spin densities at small $x, \Delta \sigma_{L}^{j e t}(s)$ grows dramatically with increase of energy if the net spin carried out by the gluons is sizable. For example, $\Delta \sigma_{L}^{j e t}(s)$ can be as large as $26 \mu b$ and 57 $\mu b$ at $\sqrt{s}=100$ and $200 \mathrm{GeV}$, respectively, whereas it is less than $1 \mu b$ if the gluons are unpolarized [47]. Of course, the behavior of the two-spin asymmetry $A_{L L}^{\text {jet }}$ is also essentially different for the cases of polarized and unpolarized gluons.

\subsubsection{Drell-Yan Processes and Polarization of Sea}

The quark spin densities can be obtained under study of the Drell-Yan production of the lepton pairs :

$$
p_{\rightarrow}+p_{\rightarrow} \rightarrow \mu^{+}+\mu^{-}+X
$$

and

$$
p_{\rightarrow}+\bar{p}_{\rightarrow} \rightarrow \mu^{+}+\mu^{-}+X
$$

The process Eq. 44 with the longitudinally polarized initial protons is extremely suitable to test the sea polarization, since $A_{L L}=0$ unless the sea quarks are polarized. 
The sign of $A_{L L}$ is opposite to that of the sea quark polarization. The expression for $A_{L L}$ has the simple form 48 :

$$
A_{L L}=-\frac{\sum_{q} e_{q}^{2}\left[\Delta q\left(x_{a}\right) \Delta \bar{q}\left(x_{b}\right)+\left(x_{a} \leftrightarrow x_{b}\right)\right.}{\sum_{q} e_{q}^{2}\left[q\left(x_{a}\right) \bar{q}\left(x_{b}\right)+\left(x_{a} \leftrightarrow x_{b}\right)\right.},
$$

where $x_{a}=\frac{1}{2}\left[x_{F}+\sqrt{x_{F}^{2}+4 s^{\prime} / s}\right]$ and $x_{b}=\frac{1}{2}\left[-x_{F}+\sqrt{x_{F}^{2}+4 s^{\prime} / s}\right]$. Hence, even measurement of the sign of $A_{L L}$ will provide information on the sea quark polarization in the polarized hadron. On the other hand the process Eq. 45 can be used to extract the polarization of valence quarks. One could expect large asymmetries in this process. However, the experimental difficulties in realization of such collisions are evident.

If the two polarized nucleons are available in the initial state then one could measure the three-spin parameters such as $(l, l, l, 0)$ in the process:

$$
p_{\rightarrow}+p_{\rightarrow}=\Lambda_{\rightarrow}+X
$$

where as always polarization of $\Lambda$-hyperon is studied through its decay process. Measurements of the three-spin correlation parameters would be important for study of the hyperon production dynamics and the strangeness content of the proton.

\subsection{4 $\chi$-Production Processes}

Number of proposals have been made to study the longitudinal and transverse spin structures of nucleons at the Relativistic Heavy Ion Collider under construction at BNL [3]. Besides the direct photons, jets and the Drell-Yan processes, the study of $J / \psi, \chi_{2}$ and $\chi_{0}$ production at RHIC has been considered. The $\chi$-production processes are interesting from the point of view of the experimental measurements of the gluon spin density [49]. The longitudinal two-spin asymmetry $A_{L L}$ for that case has the form 49, 50]:

$$
A_{L L}=a_{L L}\left(\frac{\Delta g\left(M / \sqrt{s}, M^{2}\right)}{g\left(M / \sqrt{s}, M^{2}\right)}\right)^{2}
$$

where $M$ is the invariant mass of the final state and $a_{L L}$ is the asymmetry for the subprocesses

$$
g+g \rightarrow \chi_{2} \quad\left(a_{L L}=-1\right)
$$

or

$$
g+g \rightarrow \chi_{0} \quad\left(a_{L L}=+1\right)
$$

Thus the asymmetry of inclusive $\chi_{2}$ production is a good probe of the gluon spin density. The possible experimental difficulties related to the different production mechanism contributions can be probably avoided [50].

There are also proposals to study the parity violation effects at RHIC in the decay $W^{+} \rightarrow e^{+}+\nu$ 51. 


\subsubsection{One-Spin Asymmetry Probe of Parton Spin Densities}

In the above measurements the both initial state protons are to be polarized. It can be realized in the polarized proton beam scattering on polarized target or in the collision of the two polarized proton beams. At present such experimental facilities are not available. The tertiary polarized proton beam used at Fermilab is not appropriate for that purpose because of its low intensity. Acceleration of polarized protons at Fermilab or at the UNK and the use of polarized target would provide the necessary luminosity for such hard scattering experiments in the future.

Interesting proposal to use the existing experimental facilities to study the proton spin structure has been done recently [53] (see also [52]). There the following process was considered:

$$
p_{\rightarrow}+p \rightarrow \mu^{+}+\mu^{-}+X,
$$

with one longitudinally polarized proton in the initial state when the momenta of the both outgoing muons are measured. In this case the longitudinal asymmetry $A$ for the process Eq. 51 will be proportional to the quantity

$$
\left\langle\vec{s} \cdot \vec{q}^{+} \times \vec{q}^{-}\right\rangle,
$$

where $\vec{s}$ is the proton spin and $\vec{q}^{ \pm}$denote the momenta of the outgoing muons in the c. m. s. of the colliding protons.

The asymmetry $A$ gets a non-zero value only when the outgoing muon pair has a non-zero transverse momentum $Q_{\perp}\left(Q_{\perp}=q_{\perp}^{+}+q_{\perp}^{-}\right)$. Since the one-spin asymmetry $A$ arises from the one-loop contributions, it is proportional to the QCD running coupling constant $\alpha_{s}$. However, it was shown the coefficients of $\alpha_{s}$ that originate from the parton subprocesses are not necessarily small and that makes reasonable the experimental measurements at moderate values of $Q^{2}$. Although it is supposed that perturbation theory already works, the running coupling constant is not too small to eliminate the effect.

The two parton subrocesses provide the contribution to the muon pair production:

$$
\begin{aligned}
& q+\bar{q} \rightarrow \mu^{+} \mu^{-}+g, \\
& g+q \rightarrow \mu^{+} \mu^{-}+q,
\end{aligned}
$$

where one of the incoming partons is longitudinally polarized. The asymmetry at the parton level may reach $30 \%$ 53

It is useful to have predictions at the hadron level in order to reveal the dependence of asymmetry on the gluon spin of the total proton spin and to clarify the experimental feasibility of these measurements. It appeared that at hadron level the corresponding asymmetry has significant values at $p_{L}=70$ and $400 \mathrm{GeV} / \mathrm{c}$ and depends strongly on the gluon contribution $\Delta g$ (Figs. 4, 5). The asymmetries at higher energies are decreasing (Fig. 6). The corresponding asymmetries in $p \bar{p}$ collisions are rather small $(\sim 2-4 \%)$ due to large value of the unpolarized cross-section of this 
process. Contrary to $p p$-collisions the larger values of $\Delta g$ in $\bar{p} p$-collisions lead to the smaller asymmetries. The above results have weak dependence on the specific parameterization of parton densities. The details of such calculations will be published elsewhere [54].

Thus, the measurements of the one-spin asymmetries in the muon pair production $A=\left\langle\vec{s} \cdot \vec{q}^{+} \times \vec{q}^{-}\right\rangle$, will allow one to study the gluon contribution to the proton spin. There is no need to have very high energies for such measurements and the NEPTUN experiment with polarized target at the UNK seems to be the relevant facility.

The idea of determining the polarization of an outgoing parton through the hadron distribution in the corresponding jet (jet handedness) [61] is in line with above consideration. The notion of handedness imply the measurements of the momenta of three particles from the jet and construction of pseudovector $n^{\mu}$ with the use of these momenta. When contracted with the polarization pseudovector of the outgoing parton, it provides a parity conserving term in the decay amplitude.

Thus, required experimental setup in the above cases may consist of unpolarized proton beam and polarized target or polarized proton beam colliding with unpolarized proton (or antiproton) beam or target.

\subsection{Transverse Spin Structure of Nucleon}

New possibilities are opening for measurements of the transverse spin densities of quarks if the transversely polarized proton beams available. Here the exceptional role belongs to the Drell-Yan processes with transversely polarized beams:

$$
p_{\uparrow}+p_{\uparrow} \rightarrow \mu^{+}+\mu^{-}+X,
$$

Recently, a wide discussion of the experiments on transverse asymmetries was stimulated by the new theoretical results [56]. In our consideration below we follow the above reference.

Let us remind first the definitions of parton momentum and spin densities in the infinite momentum frame. In that frame the momentum $P$ and spin $S$ of a proton are

$$
\begin{gathered}
P=p+\frac{M^{2}}{2} n, \quad P^{2}=M^{2} ; \\
S^{\mu}=S \cdot n p^{\mu}+S \cdot p n^{\mu}+S_{T}^{\mu}, \quad S^{2}=M^{2}, \quad P \cdot S=0
\end{gathered}
$$

where $n$ and $p$ are null vectors which obey the following equations: $n^{2}=p^{2}=$ $0, \quad n^{+}=p^{-}=0, \quad n \cdot p=1$. Their mass dimensions are -1 and 1 , respectively. In the particular case of the target moving in the $\hat{z}$ direction: $p=1 / \sqrt{2}(\tilde{P}, 0,0, \tilde{P}), \quad n=$ $1 / \sqrt{2}(1 / \tilde{P}, 0,0,-1 / \tilde{P})$, where the parameter $\tilde{P}$ characterizes the reference frame.

General form of the quark spin density matrix element in the leading order for the massless case $m_{q}=0$ is as follows:

$$
P_{\alpha \beta}^{q}\left(x, \lambda, S_{T}\right)=\frac{x}{2} \gamma^{\mu} P_{\mu}\left[q(x)-h_{L}^{q}(x) \lambda \gamma_{5}+h_{T}^{q}(x) \gamma_{5} \gamma^{\nu} S_{\nu T}\right]_{\alpha \beta},
$$


Figure 4: One-spin asymmetry in the muon pair production process $p_{\rightarrow}+p \rightarrow \mu^{+}+$ $\mu^{-}+X$ versus the energy of the virtual photon in the c. m. s. at $Q_{\perp}^{2}=0.4(\mathrm{GeV} / \mathrm{c})^{2}$ at $p_{L}=70 \mathrm{GeV} / \mathrm{c}$ 
Figure 5: One-spin asymmetry in the muon pair production process $p_{\rightarrow}+p \rightarrow \mu^{+}+$ $\mu^{-}+X$ versus the energy of the virtual photon in the c. m. s. at $Q_{\perp}^{2}=3(\mathrm{GeV} / \mathrm{c})^{2}$ at $p_{L}=400 \mathrm{GeV} / \mathrm{c}$ 
Figure 6: One-spin asymmetry in the muon pair production process $p_{\rightarrow}+p \rightarrow \mu^{+}+$ $\mu^{-}+X$ versus the energy of the virtual photon in the c. m. s. at $Q_{\perp}^{2}=50(\mathrm{GeV} / \mathrm{c})^{2}$ at $p_{L}=3000 \mathrm{GeV} / \mathrm{c}$ 
where $P^{\mu}$ is the proton momentum, $\lambda$ is its helicity, $S_{T}^{\mu}$ stands for the proton transverse spin as it was defined above and $\alpha$ and $\beta$ are the Dirac indices. The quantities $q(x)$, $h_{L}^{q}(x) \equiv \Delta q(x)$ and $h_{T}^{q}(x)$ denote the unpolarized, longitudinally and transversely polarized quark densities, respectively. All of them enter the density matrix with the factor of the proton momentum and in this sense the longitudinal and transverse spin densities are comparable.

For the case of deep inelastic scattering on longitudinally polarized proton the quantity $h_{L}^{q}(x)$ contributes to the structure function $g_{1}$ in the leading order. Contrary to $h_{L}^{q}(x)$ the transverse density $h_{T}^{q}(x)$ cannot be measured in deep inelastic scattering. The reason of such difference is in the different behavior of the two parts of the quark spin density matrix under the chiral transformations. The transverse part of spin density matrix is odd: it commutes with the Dirac $\gamma_{5}$, while the longitudinal contribution is anti-commuting with $\gamma_{5}$ and thus is even. The transverse density $h_{T}(x)$ measures the correlation between the left-handed and the right-handed quarks.

Being chirally invariant the electromagnetic current cannot probe the transverse part of the density matrix. Therefore it has been proposed to consider the probe [56] with different chiral properties than the electromagnetic current has. The relevant process is the Drell-Yan lepton pair production Eq. 52 where both incoming protons are polarized. The expression for the cross-section of the process Eq. 52 in the one-photon approximation is determined by the hadronic tensor $W_{\mu \nu}$, namely:

$$
\frac{d \sigma}{d Q^{2} d y d \Omega}=\frac{\alpha^{2}}{2(2 \pi)^{4} s Q^{2}}\left(\delta_{i j}-\frac{l_{i} l_{j}}{\overrightarrow{l^{2}}}\right) W_{i j},
$$

where $l$ is the lepton momentum, $Q^{2}$ is the mass squared of the lepton pair and $y$ is its rapidity. The $d \Omega$ denotes the solid angle of $\vec{l}$ in the dimuon rest frame where the right-hand side is to be evaluated. The hadron tensor $W_{\mu \nu}$ is defined by

$$
W_{\mu \nu} \equiv e^{-2} \int d^{4} \xi\left\langle P_{A} S_{A} P_{B} S_{B}\left|J_{\mu}(0) J_{\nu}(\xi)\right| P_{A} S_{A} P_{B} S_{B}\right\rangle,
$$

where $J_{\mu}$ is the electromagnetic current and $\left(P_{A} S_{A}\right)$ and $\left(P_{B} S_{B}\right)$ are the momenta and spins of the hadrons $A$ and $B$.

In the one-photon approximation the Drell-Yan process includes the $q \bar{q}-$ pair annihilation described by the matrix element between the quark states:

$$
\left\langle k s \bar{k} s^{\prime}\left|J_{\mu}(0) J_{\nu}(\xi)\right| k s \bar{k} s^{\prime}\right\rangle \rightarrow\left\langle k s\left|q_{\alpha}(\zeta) J_{\mu}(0) J_{\nu}(\xi) \bar{q}_{\beta}(\chi)\right| k s\right\rangle .
$$

Thus, both the electromagnetic current and the antiquark field are the probes of the quark state. A transversely polarized quark can be regarded as a superposition of the longitudinally polarized states. Since antiquark interacts with quark conserving helicity and the antiquark field appears in both (even and odd) chirality combinations, this field can be used to probe the even (longitudinal) and odd (transverse) parts of the quark spin density matrix. Of course, the above statement can be reversed and the quark field can be considered as a probe of antiquark state. 
For the transverse spin density the expression in terms of the matrix element of the bilocal operator has been obtained [55]:

$$
2 S_{T}^{\mu} h_{T}^{q}\left(x, Q^{2}\right)=\frac{1}{2 \pi} \int d \xi e^{i \xi x}\left\langle P S\left|\bar{q}(0) i \sigma^{\mu \nu} n_{\nu} \gamma_{5} q(\xi n)\right| P S\right\rangle,
$$

where $\sigma_{\mu \nu} \equiv \frac{i}{2}\left[\gamma_{\mu}, \gamma_{\nu}\right]$. Eq. 59 was obtained in the infinite momentum frame and it represents a generic form for the field-theoretical definition of the parton densities. The expression for $h_{L}^{q}\left(x, Q^{2}\right)$ has the similar form:

$$
2 \lambda h_{L}^{q}\left(x, Q^{2}\right)=\frac{1}{2 \pi} \int d \xi e^{i \xi x}\left\langle P S\left|\bar{q}(0) \gamma_{5} \gamma_{\mu} n^{\mu} q(\xi n)\right| P S\right\rangle .
$$

The OPE analysis of the function $h_{T}^{q}(x)$ determined as a matrix element of the twist-two operator has been performed [55]. As it was stressed, the quantity connected with the transverse quark density $h_{T}^{q}(x)$, differs from the transverse spin operator $\Sigma_{\perp}$ by the $\gamma_{0}$ factor. Therefore, in general, for the case of off-shell particle the eigenstate of the transversity operator $\gamma_{0} \Sigma_{\perp}$ is not that of the transverse spin operator. The transverse spin operator does not commute with the free-quark Hamiltonian and depends on the underlying dynamics 60]. Therefore it was proposed to name $h_{T}^{q}(x)$ as transversity.

It is worth to note here that $\Delta_{\perp} q(x)$ and $h_{T}^{q}(x)$ are different quantities in the parton model. The $\Delta_{\perp} q(x)$ measures an average transverse spin of quarks while the quantity $h_{T}^{q}(x)$ measures the net number of quarks in the transversity eigenstate [56], [59.

There were considered also the higher-twist transverse structure functions which do not allow simple partonic interpretations and are potentially useful for the studies of nonperturbative effects in QCD.

It should be noted that quarks and antiquarks contribute to $h_{L}^{q}(x)$ and $h_{T}^{q}(x)$ in different ways. The sum rule for the function $h_{T}^{q}(x)$

$$
\int_{0}^{1} d x\left[h_{T}^{q}(x)-h_{T}^{\bar{q}}(x)\right]=\delta q
$$

shows that contrary to $\Delta q$ the "tensor charge" $\delta q$ gets contributions from the valence quarks only. There is no gluon contribution to $h_{T}^{q}(x)$ due to the angular momentum conservation [56]. Contrary to $h_{L}^{q}$ it evolves with $Q^{2}$ uncoupled with the gluon density. Therefore the ambiguities related to the gluon anomaly are not to be present here.

\subsubsection{Experiments to Measure the Transverse Spin Quark Densities}

The quantities $h_{T}^{q}(x), h_{L}^{q}(x)$ along with the unpolarized quark density $q(x)$ determine the angular distribution of lepton pairs in the Drell-Yan process [57:

$$
\frac{d \sigma}{d Q^{2} d y d \Omega}=\quad \sum_{q} \frac{\alpha^{2} e_{q}^{2}}{12 Q^{2} s}\left\{\left[q^{A}\left(x_{A}\right) \bar{q}^{B}\left(x_{B}\right)-\lambda_{A} \lambda_{B} h_{L}^{q A}\left(x_{A}\right) h_{L}^{\bar{q} B}\left(x_{B}\right)\right] \times\right.
$$




$$
\begin{aligned}
& \left(1+\cos ^{2} \theta\right)+S_{T A} S_{T B} h_{T}^{q A}\left(x_{A}\right) h_{T}^{\bar{q} B}\left(x_{B}\right) \sin ^{2} \theta \times \\
& \left.\cos \left(2 \varphi-\varphi_{A}-\varphi_{B}\right)+A \leftrightarrow B\right\},
\end{aligned}
$$

where $e_{q}$ is the quark electric charge in units of the proton charge. The $Q^{2}$-dependence is implied for the quark densities. In Eq. $62 \theta$ is the polar angle and $\varphi$ is the azimuthal angle both related to the beam direction; $\varphi_{A}$ and $\varphi_{B}$ denote the azimuthal angles of the transverse spin projections for the nucleons $A$ and $B$. From the angular distribution Eq. 62 an asymmetry $\Delta \sigma$ which depends on the transverse densities alone can be obtained by means of integration over all the polar angles and subtraction of the integrals over the azimuthal angle $\varphi$ :

$$
\frac{d \Delta \sigma}{d Q^{2} d y}=\int_{\pi / 4}^{3 \pi / 4}+\int_{5 \pi / 4}^{7 \pi / 4}-\int_{3 \pi / 4}^{5 \pi / 4}-\int_{-\pi / 4}^{\pi / 4} d \varphi \frac{d \sigma}{d Q^{2} d y d \varphi}
$$

where the spins of nucleons $S_{T A}$ and $S_{T B}$ are chosen to be oriented along $\hat{x}$ axis $\left(\varphi_{A}=\varphi_{B}=0\right)$. The quantity Eq. 63 in terms of the transverse quark densities is

$$
\frac{d \Delta \sigma}{d Q^{2} d y}=\frac{4}{9} \sum_{q} \frac{\alpha^{2} e_{q}^{2}}{Q^{2} s} h_{T}^{q A}\left(x_{A}\right) h_{T}^{\bar{q} B}\left(x_{B}\right) S_{T A} S_{T B}+A \leftrightarrow B .
$$

Thus, the measurements of lepton pair angular distribution in the Drell-Yan process will allow to determine the transverse spin densities for quarks and antiquarks. Until recently the transverse spin densities have been almost ignored in deep inelastic scattering processes because of their higher-twist origin. Fortunately, the recent progress in that field made clear the necessity of the experimental studies of these densities in the Drell-Yan processes. This is completely unexplored domain of spin physics.

Besides the Drell-Yan processes there are several possibilities to study the transverse quark densities [56]. One could mention the process of $\pi^{+} \pi^{+}$-pair production

$$
p_{\uparrow}+p_{\uparrow} \rightarrow \pi^{+}+\pi^{+}+X
$$

dominated by the scattering of the two $u$-quarks at large and opposite $p_{\perp}$ values and the semi-inclusive deep inelastic scattering reaction

$$
e^{-}+p_{\uparrow} \rightarrow e^{-}+\Lambda_{\uparrow}+X
$$

Since polarization of $\Lambda$ can be measured through its decay, the measurements of the parameter $D_{N N}$ will allow to restore the transverse spin densities of quarks.

In the recent paper [58] it was shown that the transverse spin of quark initiating a jet can be measured through the azimuthal dependence of pion pairs in the jet fragments.

There is also good chance to study the transverse spin densities of quarks in the direct $\gamma$ production in the polarized $p \bar{p}$ collisions. Contrary to the longitudinally polarized case, only quark annihilation will contribute to the asymmetry in the direct $\gamma$ production since gluons cannot be polarized transversely in the spin $-1 / 2$ nucleon. At favorable conditions the values of asymmetries can reach $10-20 \%$ [59. The measurements of the two-spin asymmetries $A_{N N}$ in the production of heavy quark pairs will also supply the data on the transverse spin quark densities. 


\section{Spin Phenomena and Electroweak Interactions}

It should be noted that the spin effects expected under the searches and exploration of new objects, should be observed in the presence of intensive hadronic processes. Therefore, to separate such effects one is to be able to calculate the hadronic asymmetries. They require in their turn the knowledge of the corresponding quark and gluon spin densities. Till now, the information about these densities is rather fragmentary. The perspectives for the experimental studies of the longitudinal and transverse spin densities will be discussed further. Their knowledge is also vital for variety of the calculations in electroweak sector of the Standard Model based on the $S U(2)_{L} \times U(1)_{Y}$ gauge symmetry. There are four gauge bosons associated with this symmetry: the well known $W^{ \pm}-, Z^{0}$-bosons and photon. The subscript $L$ reminds that the charge changing weak interactions describe transitions between left-handed fermions only (i.e. they violate parity conservation) while the $\mathrm{Z}$ coupling to a fermion is a mixture of the pure $(V-A)$ coupling of $S U(2)_{L}$ and the vector coupling of QED.

In electroweak interactions, where the parity is not conserved, there could be observed significant one-spin longitudinal asymmetries as well as transverse ones. Let us consider the case of $W^{+}$- or $W^{-}$-boson production. The amplitudes of the corresponding processes

$$
\begin{aligned}
& p_{\rightarrow}+\bar{p} \rightarrow W^{+}+X \\
& p_{\rightarrow}+\bar{p} \rightarrow W^{-}+X
\end{aligned}
$$

may be calculated by means of account for the contributions of the dominant fusion reactions only: $u+\bar{d} \rightarrow W^{+}$or $\bar{u}+d \rightarrow W^{-}$. For the case of longitudinally polarized proton the asymmetry at the level of the subprocess will be maximal $\left(a_{L}=100 \%\right)$ since $\mathrm{W}$ is the left-handed current. The asymmetry at the hadronic level $A_{L}$ will also become essential after $a_{L}$ is integrated with the quark spin densities $\Delta q_{i}\left(x, Q^{2}\right)$. In the above approximation to the Drell-Yan mechanism the asymmetry $A_{L}$ is an algebraic combination of the quark densities. The values of $A_{L}$ in the above-mentioned reactions can reach $60-80 \%$. These asymmetries decrease very slowly with the energy. Apart from the independent test of electroweak sector of the Standard Model, the experimental studies of such a dependence allow to get information on the quark spin densities.

Due to the chiral properties of $W$-boson the asymmetry at the level of the subprocess $a_{L}$ will be universal for different pairs of quarks $q_{i}$ and $q_{j}$ in the processes

$$
q_{i}+\bar{q}_{j} \rightarrow W^{ \pm}(M) \rightarrow X
$$

where the final state $X$ contains $W^{ \pm}$and a neutral particle ( $M$ is the invariant mass of the final state particles).

Similar universality will characterize the asymmetries $A_{L}$ in those hadronic processes where the contributions from the subprocesses Eq. 67 are dominant. The 
following reactions are particular examples of such hadronic reactions:

$$
p+p \rightarrow W^{ \pm}+\gamma, W^{ \pm}+Z^{0}, W^{ \pm}+H^{0} .
$$

The details can be found in extensive review [24].

The minimal extension of the Standard Model consists in introduction of the models with the left-right symmetry when the parity violation is considered as a low-energy effect. These models imply the existence of the right-handed $W$-bosons. The asymmetries in the production of $W_{R}^{+}$- and $W_{R}^{-}$-bosons are directly obtained from the corresponding asymmetries in the production of left $W$-bosons by means of reversal of the asymmetry signs owing to the change of sign of the axial coupling constant.

The collisions of polarized proton beam with the unpolarized one could provide an additional information on a possible strong electroweak sector. Recently spin asymmetries were calculated for that purpose using BESS model (Breaking the Electroweak Symmetry Strongly) [63]. Here the spin asymmetries different from those predicted by the Standard Model could indicate the presence of the strong electroweak sector. BESS model predicts the vector resonances (V-particles) which are bound states of a strongly interacting sector. The measurements of the one-spin asymmetry $A_{L}$ in the processes

$$
p_{\rightarrow}+p(\bar{p}) \rightarrow l^{+} l^{-}+X
$$

could allow to distinguish $V^{ \pm}$bosons from $W_{R}$ boson in the region where $q \bar{q}$-processes dominate. Thus, it allows one to discriminate between the different models of electroweak interactions.

\section{Search for New Particles and Spin Effects}

\subsection{Compositeness}

Polarization measurements may also be useful for study of the compositeness. Currently, a large number of models treat quarks and leptons as the composite particles. The interaction between the new constituents (preons) may generate the interactions with an arbitrary chiral structure which can violate the parity conservation.

The both $A_{L}$ and $A_{N}$ measurements are useful for search of the compositeness. The simplest signal for the quark compositeness is deviation of the jet production cross section at large transverse momenta from the values predicted by perturbative QCD. This deviation would arise from the new interaction between quarks induced by their composite structures

$$
\mathcal{L}=\mathcal{L}_{Q C D}+\eta_{0} \frac{g^{2}}{\Lambda_{c}^{2}} \bar{q} A q \bar{q} A q,
$$

where $\Lambda_{c}$ is the scale of the compositeness of the order of the binding energy for preons (it is usually taken to be $\sim 1-2 \mathrm{TeV}$ ), $A$ determines the Dirac structure of interaction 
and depends on the details of particular composite model. As it is generally believed the new interactions induce a parity violating term in the lagrangian. The above mentioned deviation from QCD-interactions may be found while measuring the onespin longitudinal asymmetry $A_{L}$ in hard hadronic processes. In virtue of the parity violation in the preon interaction, this quantity will differ from zero and become rather large at $p_{\perp} \simeq 3-4 \mathrm{GeV} / c$. Therefore, the large longitudinal asymmetries in hadron interactions may occur due to manifestation of the preon interactions. Fig. 7 presents the calculations [64 for the asymmetry $A_{L}$ in the jet production in hard $p p$-interactions.

Noticeable effects will be also observed under production of direct photons or lepton pairs. The compositeness should enlarge polarization effects in these reactions too.

The important role of the parallel studies of the polarization and the compositeness consists in providing the opportunities for the choice of particular type of the interaction, i.e. the form of $A$. Indeed, the different forms of $A$ such as $A=\gamma^{\mu}\left(1-\gamma_{5}\right) / 2$ or $A=\gamma^{\mu}$ provide almost the same predictions for the spin-averaged cross sections [65. On the other hand predictions for the $A_{L}$ parameter are essentially different for the above two options of $A$.

\subsection{Supersymmetry}

The SUSY theories predict the existence of a series of new particles. The supersymmetric partners of ordinary particles are known to differ by $1 / 2$ unit in their spins. Therefore, the asymmetries appearing in the production of SUSY particles in the polarized hadron collisions, will differ from the corresponding asymmetries arising in the production of ordinary particles.

Since light SUSY particles interact with ordinary matter weakly, the production of supersymmetric particles is characterized by the events with the missing energymomentum. It is difficult to interpret such events; that is why polarization measurements may turn out to be quite useful. For instance, in the strong sector one should expect production of a large amount of scalar quarks (squarks, spin 0) and gluinos (spin 1/2). A characteristic feature of this type events is the jet production and the "missing energy". Since in strong interactions the parity is conserved one should consider the two-spin asymmetries $A_{L L}$ as a relevant spin parameter. For the processes, where a pair of SUSY particles is produced in the final state, the subprocess asymmetry $a_{L L}^{i j}=-100 \%$ for the case of massless squarks and gluinos because of the helicity conservation. As a result, the double asymmetry $A_{L L}$ should be negative and have larger values than in the case of the ordinary particles. To illustrate this statement Figs. 8, $a$ and 8,b represent the predictions for the parameter $A_{L L}$ in the two cases of the ordinary and supersymmetric particle production respectively [24]. Therefore, the most typical feature of the SUSY particle production is appearance of the events with specific behavior of the polarization asymmetries. 
Figure 7: The asymmetry $A_{L}$ in reaction $p p \rightarrow j e t+X$ 
Figure 8: The asymmetry $A_{L L}$ for production of the ordinary particle $(a)$ and supersymmetric particle $(b)$ 


\section{$5 \quad$ Spin Effects in $e^{+} e^{-}-$Collisions}

Electron-positron collisions are highly interesting since the spin effects are related to the interactions at small distances. This is due to the fact that $e^{+} e^{-}$-interactions are mediated by the electroweak currents $\left(\gamma, Z, W^{ \pm}, \ldots\right)$ which have point-like couplings and a simple spin structure. $e^{+} e^{-}$-interactions allow also high accuracy measurements. The main goal of any $e^{+} e^{-}$-collider program is the physics of $Z^{0}$-and $W^{ \pm}$-bosons. The interest in the respective polarization studies of these processes lies mainly in the high energy region, where weak interactions become especially important.

It should be noted that the radiation effects lead to the appearance of a natural transverse polarization in $e^{+} e^{-}$storage rings

$$
P_{\perp}\left(e^{+}\right)=-P_{\perp}\left(e^{-}\right)
$$

which may exceed $90 \%$. It should be noted that the transverse polarization leads to a certain azimuthal dependence that was used to determine the beam polarization and to verify the fermionic nature of quarks.

In $e^{+} e^{-}$-collisions, the colliding particles produce a virtual intermediate state that, in accordance with the Standard Model, is either a photon or $Z^{0}$-boson. In this case, the amplitudes are completely defined by the coupling constants of the gauge bosons $\left(\gamma\right.$ or $\left.Z^{0}\right)$ with the final states. Therefore, spin effects in these reactions depend on the initial spins only, whereas angular asymmetries are related to direction of the beam polarization.

The dependence of the $e^{+} e^{-}$-annihilation cross sections on the beam polarization appears due to interference of the contributions from the virtual $\gamma$ and $Z^{0}$. Then, since at the energies higher than $200 \mathrm{GeV}$ the value of the contribution from weak interactions is comparable with that of electromagnetic interactions, one should expect significant polarization effects. In this energy range the asymmetry $A_{L}$

$$
A_{L}=\frac{\sigma(+-)-\sigma(-+)}{\sigma(+-)+\sigma(-+)}=\frac{\sigma(+0)-\sigma(-0)}{\sigma(+0)+\sigma(-0)}=\frac{\sigma(0+)-\sigma(0-)}{\sigma(0+)+\sigma(0-)}
$$

(by the signs,+- and 0 we mean the longitudinal polarization $P_{L}=+1,-1$ and 0 , respectively) has the following values [24 for the different processes:

$$
\begin{aligned}
& e^{+} e^{-} \rightarrow \quad e^{+} e^{-} \quad-\quad 7 \%, \\
& \rightarrow \nu \bar{\nu}-15 \%, \\
& \rightarrow u \bar{u}, c \bar{c}, t \bar{t}-34 \% \text {, } \\
& \rightarrow d \bar{d}, s \bar{s}, b \bar{b}-62 \%, \\
& \rightarrow \quad Z^{0} Z^{0}-32 \% \text {, } \\
& \rightarrow W^{+} W^{-}-94 \% \text {. }
\end{aligned}
$$




\begin{tabular}{||c|c|c|c|c||}
\hline \hline & $\nu$ & $e$ & $u$ & $d$ \\
\hline \hline $2 \sin \theta_{W} a_{f}$ & 1 & $-1+4 \sin ^{2} \theta_{W}$ & $1-\frac{8}{3} \sin ^{2} \theta_{W}$ & $-1+\frac{4}{3} \sin ^{2} \theta_{W}$ \\
\hline $2 \sin \theta_{W} b_{f}$ & 1 & -1 & 1 & -1 \\
\hline \hline
\end{tabular}

Table 1: Axial and vector coupling constants for leptons and quarks, $\theta_{W}$ is the Weinberg angle

Verification of these values would provide an important test of the Standard Model when the initial $Z$-fermion-antifermion vertex

$$
-i e \bar{u}_{f} \gamma^{\mu}\left(a_{f}-b_{f} \gamma^{5}\right) u_{f}
$$

is determined by the vector and axial coupling constants. The corresponding values for leptons and quarks are given in the Table 1.

Beyond the Standard Model, investigations of spin effects might be used for the search of the new physics. Thus, for instance, the $Z^{\prime}$-boson, predicted in the Grand Unification Theories should manifest itself as a peak in the $e^{+} e^{-}$-interaction cross section. If so, even out of the resonance region one would observe a considerable deviation of the asymmetry $A_{L}$ from the behavior predicted by the Standard Model. The measurements of such deviation would allow to extract the coupling constants for the interactions of $Z^{\prime}$ with different fermions.

In $e^{+} e^{-}$-interactions the polarization effects may be essential for the search for the SUSY particles. Both longitudinally and transversely polarized electron beams are equally important for these purposes.

In conclusion it should be noted that spin physics experiments will provide the crucial tests for the present theories and undoubtedly will bring new unexpected results. This justifies the efforts needed to perform the spin measurements and to create the new spin physics facilities.

We would like to thank A. D. Krisch, D. I. Patalakha, J. P. Ralston and V. L. Solovianov for useful discussions, comments and suggestions.

\section{References}

[1] A. D. Krisch, Phys. Rev. Lett. 63 (1989) 1137.

[2] SPIN Collaboration (Progress Report), University of Michigan (Ann Arbor, 1992)

[3] G. Bunce at al., Particle World 3 (1992) 1.

[4] Proposal on Spin Physics Using the RHIC Polarized Collider, 14 August 1992. 
[5] HERMES Collaboration, A Proposal to Measure the Spin-Dependent Structure Functions Neutron and Proton at HERA ( DESY, 1990).

[6] G. L. Kane, J. Pumplin and W. Repko Phys. Rev. Lett. 41 (1978) 1989.

[7] G. L. Kane, G. A. Ladinsky and C.-P. Yuan, Using the Top Quark for Testing Standard Model Polarization and CP Predictions, SSCL-486, 1991.

[8] J. Qiu and G. Sterman, Nucl. Phys. B 353 (1991) 105;

[9] S. J. Brodsky and G. P. Lepage, Phys. Rev. D24 (1981) 2848.

[10] S. M. Troshin and N. E. Tyurin, Int. Journ. of Mod. Phys. A 5 (1990) 2689;

[11] J. P. Ralston and B. Pire, Preprint Kansas 5-15-92, 1992.

[12] P. V. Landshoff, Phys. Rev. D10 (1974) 1024.

[13] A. H. Mueller, Phys. Rep. 73 (1981) 237.

[14] J. Botts and G. Sterman, Nucl. Phys. B325 (1989) 62.

[15] A. D. Krisch, in Proceedings Workshop "Physics at UNK" Eds. S. I. Bityukov and A. M. Zaitsev (IHEP, Protvino, 1989) .

[16] A. D. Krisch, in Proceedings of the 7th International Symposium on High Energy Spin Physics, Ed. L. D. Soloviev (IHEP, Serpukhov, 1987).

[17] V. L. Solovianov in Proceedings Workshop "Physics at UNK" Eds. S. I. Bityukov and A. M. Zaitsev (IHEP, Protvino, 1989) .

[18] J. P. Ralston and B. Pire, Phys. Rev. Lett. 65 (1990) 2343.

[19] A. V. Efremov and O. V. Teryaev, Yad. Fiz. 36 (1982) 242 [ Sov. J. Nucl. Phys. 36 (1982) 140].

[20] J. Qiu and G. Sterman, Phys. Rev. Lett. 67 (1991) 2264.

[21] X. Ji, Phys. Lett. B 289 (1992) 137.

[22] S. J. Brodsky, P. Hoyer, A. H. Mueller and W.-K. Tang, Nucl. Phys. B369 (1992) 519.

[23] N. S. Craigie, K. Hidaka, M. Jacob and F. M. Renard, Phys. Rep. 99 (1983) 69.

[24] C. Bourelly, J. Soffer, F. M. Renard and P. Taxil, Phys. Rep. 177 (1989) 319.

[25] L. Pondrom, in Proceedings of the 7th International Symposium on High Energy Spin Physics, Ed. L. D. Soloviev (IHEP, Serpukhov, 1987) .

[26] J. Ashman at al., Nucl. Phys. B238 (1989) 1. 
[27] E. V. Shuryak, The QCD Vacuum, Hadrons and Superdense Matter (World Scientific, Singapore, 1988)

[28] S. M. Troshin and N. E. Tyurin, Phys. Lett. B 144 (1984) 260;

S. M. Troshin and N. E. Tyurin, Spin Effects in Hard Interactions (Interest in Accelerated Polarized Beam) IHEP 92-76, 1992;

S. M. Troshin and N. E. Tyurin, Nuovo Cim. A, (1993) to be published.

[29] L. A. Ahrens et al., Phys. Rev. D 35 (1987) 785.

[30] D. B. Kaplan and A. V. Manohar, Nucl. Phys. B 310 (1988) 527.

[31] C. S. Lam and Bing-An Li, Phys. Rev. D25 (1982) 683;

A. V. Efremov and O. A. Teryaev, JINR Preprint E2-88-287 (1988) Dubna;

G. Altarelli and G. G. Ross, Phys. Lett. B212 (1988) 391;

R. D. Carlitz, J. C. Collins and A. H. Mueller, Phys. Lett. B214 (1988) 229.

[32] F. J. Yndurain, Quantum Chromodinamics (Springer Verlag, New-York, 1983).

[33] J. Kodaira, Nucl. Phys. B165 (1980) 129.

[34] R. L. Jaffe and A. Manohar, Nucl. Phys. B337 (1990) 509.

[35] A. V. Kisselev and V. A. Petrov, IHEP Preprint 91-171 (1990).

[36] S. Forte, Nucl. Phys. B331 (1990) 1.

[37] G. Preparata, Invited talk at Adriatico Research Conference "Polarization Dynamics in Nuclear and Particle Physics", Trieste, 1992.

[38] A. V. Manohar, in Proceedings Polarized Collider Workshop, Eds. J. Collins, S. F. Heppelman and R. W. Robinett (AIP, New York, 1991).

[39] J. D. Bjorken, Phys. Rev. 148 (1966) 1467.

[40] R. L. Jaffe and X. Ji, Phys. Rev. D43 (1991) 724.

[41] M. Anselmino and E. Leader, Phys. Lett. B293 (1992) 216.

[42] W. Wandzura and F. Wilczek, Phys. Lett. B172 (1977) 195.

[43] J. D. Jackson, G. G. Ross and R. G. Roberts, Phys. Lett. B 226 (1989) 159.

[44] H. Burkhardt and W. N. Cottingham, Ann. Phys. (N. Y. ) 56 (1970) 453.

[45] O. Chamberlain, in Polarized Beams at SSC, Eds A. D. Krisch, A. M. T. Lin and O. Chamberlain (AIP, New York, 1986) .

[46] E. L. Berger and J. Qiu, in Proceedings Polarized Collider Workshop, Eds. J. Collins, S. F. Heppelman and R. W. Robinett (AIP, New York, 1991). 
[47] J. Qiu, G. P. Ramsey, D. Richards and D. Sivers, ANL Preprint PR 88-52 (1988).

[48] F. Close and D. Sivers, Phys. Rev. Lett. 39 (1977) 1116.

[49] J. L. Cortes and B. Pire, Phys. Rev. D 38 (1988) 3586.

[50] M. A. Doncheski, in Proceedings Polarized Collider Workshop, Eds. J. Collins, S. F. Heppelman and R. W. Robinett (AIP, New York, 1991) .

[51] M. J. Tannenbaum, in Proceedings Polarized Collider Workshop, Eds. J. Collins, S. F. Heppelman and R. W. Robinett (AIP, New York, 1991) .

[52] B. Pire and J. P. Ralston, Phys. Rev. D 28 (1983) 260.

[53] R. D. Carlitz and R. S. Willey, Phys. Rev. Lett. D45 (1992) 2323.

[54] P. M. Nadolsky, to be published.

[55] E. Shuryak and A. Vainshtein, Nucl. Phys. B 201 (1982) 141;

A. V. Efremov and O. A. Teryaev, Sov. J. Nucl. Phys. 39 (1984) 962.

[56] J. P. Ralston and D. E. Soper, Nucl. Phys. B152 (1979) 109;

X. Artru and M. Mekhfi, Z. Phys. C 45 (1990) 669;

R. L. Jaffe and X. Ji, Phys. Rev. Lett. 67 (1991) 552.

[57] J. L. Cortes, B. Pire and J. P. Ralston, in Zeit. Phys. C 55 (1992) 409.

[58] J. C. Collins, S. F. Heppelmann and G. A. Ladinsky, Preprint PSU/TH/101, April 6, 1993.

[59] X. Ji, Phys. Lett. B 284 (1992) 137.

[60] J. Kogut and D. E. Soper, Phys. Rev. D1 (1970) 2901.

[61] O. Nachtmann, Nucl. Phys. B 127 (1977) 314;

A. V. Efremov, L. Mankiewich and N. A. Tornqvist, Phys. Lett. B284 (1992) 394.

[62] M. Glück and W. Vogelsang, Phys. Lett. B277 (1992) 515;

A. P. Contogouris and S. Papadoupolous, Phys. Lett. B260 (1991) 204; W. Vogelsang and A. Weber, Preprint Do Th 92-26, Dortmund, 1992.

[63] R. Casalbuoni at al., Phys. Lett. B 279 (1992) 397;

[64] P. Taxil, in Proceedings of the 8th Intern. Symp. on High Energy Spin Physics, Ed. K. Heller (AIP, New York, 1989).

[65] I. Hinchliffe, in Proceedings of the 1984 Summer Study on the Design and Utilization of the Superconducting Super Collider, Ed. R. Donaldson and J. G. Morfin. 